ज्ञ FRANÇAISE

$\supset \mathrm{DE}$

딜 PEAGOGIE

\section{Revue française de pédagogie}

Recherches en éducation

155 | avril-juin 2006

La motivation scolaire : approches récentes et perspectives pratiques

\title{
Les évolutions du travail enseignant en France et en Europe : facteurs de changement, incidences et résistances dans l'enseignement secondaire
}

Changes in the teachers' work in France and in Europe. The elements responsible for the change, its effects and the opposition of high school teachers

Las evoluciones del trabajo de docente en Francia y en Europa: factores de cambio, incidencias y resistencias en la enseñanza secundaria

Die Entwicklung der Lehrarbeit in Frankreich und in Europa :

Veränderungsfaktoren, Folgen und Widerstandsformen in der Sekundarstufe

\section{Christian Maroy}

\section{(2) OpenEdition \\ Journals}

\section{Édition électronique}

URL : http://journals.openedition.org/rfp/273

DOI : $10.4000 /$ rfp. 273

ISSN : 2105-2913

\section{Éditeur}

ENS Éditions

Édition imprimée

Date de publication : 1 juin 2006

Pagination : 111-142

ISBN : 978-2-7342-1047-4

ISSN : 0556-7807

\section{Référence électronique}

Christian Maroy, «Les évolutions du travail enseignant en France et en Europe : facteurs de changement, incidences et résistances dans l'enseignement secondaire », Revue française de pédagogie [En ligne], 155 | avril-juin 2006, mis en ligne le 21 septembre 2010, consulté le 30 avril 2019. URL : http://journals.openedition.org/rfp/273; DOI : 10.4000/rfp.273 


\title{
NOTE DE SYNTHÈSE
}

\section{Les évolutions du travail enseignant en France et en Europe : facteurs de changement, incidences et résistances dans l'enseignement secondaire (1)}

Christian Maroy

\begin{abstract}
Face à une école et à une société en transformation rapide, cet article présente une synthèse de ce qu'on sait de l'évolution effective du travail des enseignants du secondaire. Le travail prescrit aux enseignants s'infléchit de façon convergente en Europe: les enseignants doivent, outre leurs tâches traditionnelles de préparation de cours, d'enseignement et d'évaluation, participer davantage à la vie de leur école, s'engager dans un travail collectif, se former... Au niveau du travail réel, la littérature en sciences de l'éducation s'accorde sur un constat d'intensification et de complexification du travail des enseignants. L'intensification se marque moins par un allongement de la durée du travail que par un alourdissement et une extension des tâches à réaliser et par une complexification du travail en classe. Cependant, cette intensification du travail ne doit pas seulement être rapportée à des évolutions du public scolaire mais aussi, dans les pays anglo-saxons, aux politiques scolaires basées sur l'évaluation et l'accountability. En conclusion, hous nous interrogeons sur les raisons de la tiédeur pour ne pas dire de la résistance des enseignants, face à des réformes scolaires qui sont supposés les aider. Le problème est-il seulement celui d'une résistance au changement liée à des cultures professionnelles dépassées ? Le malaise enseignant nous semble plus profondément lié à des formes de retrait ou d'opposition aux réformes, lorsqu'elles accentuent la déprofessionnalisation des enseignants.
\end{abstract}

Descripteurs (TEE) : conditions de travail de l'enseignant, développement de l'enseignement, enseignement secondaire, Europe, professionnalisation. 


\section{INTRODUCTION}

Dans la plupart des pays capitalistes « avancés ", les sociétés et l'institution scolaire sont en proie à des transformations importantes. Des transformations économiques de nature structurelle (post-fordisme, globalisation, " nouvelle économie du savoir") favorisent le développement de nouvelles demandes de dirigeants économiques pour faire face au nouveau contexte de compétition économique (développement du niveau général de "compétences" de la population active, efficacité et efficience des systèmes d'éducation et de formation, "adéquation » des qualifications). Par ailleurs, des transformations sociales (exclusion sociale et inégalités croissantes ; inquiétude des classes moyennes face à l'avenir professionnel et social de leurs enfants) favorisent la montée de demandes diverses, en tension, accentuant tantôt la nécessité de lutter contre l'exclusion sociale et les inégalités, tantôt d'améliorer la qualité et la diversité du système pour répondre à l'inquiétude des classes moyennes. Dans le même temps le processus de démocratisation quantitative des systèmes d'enseignement secondaire et supérieur continue et augmente l'hétérogénéité des publics scolaires et des situations éducatives. Les transformations culturelles (individualisation accrue, transformation du rapport à la norme et à l'autorité) favorisent en outre l'éclosion d'une demande de diversification et d'individualisation des parcours et des méthodes d'enseignement pour faire droit à la diversité des "projets ", " difficultés " et/ou situations des jeunes. Enfin, last but not least, le discours néolibéral tend à interroger les formes " bureaucratiques " d'intervention de l'État et génère outre une critique du niveau des dépenses publiques, la valorisation de modèles de gestion et de gouvernance des systèmes qui fassent droit à des méthodes et valeurs naguère surtout présents dans l'univers économique privé.

Ces transformations sont inter-reliées et peuvent affecter directement l'enseignant dans sa classe (qu'il s'agisse par exemple des effets de la " massification " de l'enseignement ou de l'évolution du rapport à l'autorité). Cependant, les enseignants sont confrontés aussi aux conséquences des politiques de réforme importante des systèmes scolaires qui sont initiées dans de nombreux pays pour " adapter " les systèmes scolaires à ces nouvelles "donnes" sociales, économiques et culturelles (Whitty et al., 1998 ; Maroy, à paraitre). Sans vouloir ici rendre compte de ces politiques, on peut penser qu'on assiste à des changements des modes de régulation des systèmes d'éducation qui se développent sur trois plans au moins : le « pilotage " du système, la conception de l'établissement et la professionnalité des enseignants (Maroy, 2002d). La modernisation de l'école passerait ainsi par la promotion d'un régime de gouvernance et de régulation post-bureaucratique (mélange variable de recours au modèle de l'État évaluateur et parfois du quasi marché ; voir Maroy, à paraître), l'attribution de plus d'autonomie à un établissement supposé devenir mobilisé - autour de sa direction favorisant le travail d'équipe et l'éclosion d'un «projet " (Dupriez, 2002) - et enfin, la " professionnalisation " et la conversion progressive des enseignants à de nouveaux modèles de professionnalité, le modèle d'un enseignant pédagogue et/ou «praticien réflexif » (Cattonar \& Maroy, 2000) (2).

Le discours modernisateur qui sous-tend nombre de politiques éducatives pourrait donc être abruptement résumé de la façon suivante. Grâce à des établissements plus autonomes, développant des projets éducatifs portés par des enseignants engagés dans une dynamique collective, grâce à des enseignants pédagogues, réflexifs et centrés sur l'apprentissage de l'élève, grâce aussi à un cadrage institutionnel où l'État régule et évalue les unités d'enseignement décentralisées, l'école devrait pouvoir affronter les défis auxquels elle est confrontée. Elle devrait devenir simultanément plus juste et plus efficace. 
C'est dans ce contexte très général, et trop rapidement brossé, que nous voudrions traiter des évolutions du travail enseignant et de quelques enjeux qu'elles soulèvent, en nous basant sur une synthèse partielle des connaissances les plus solides de la littérature en sciences de l'éducation.

Dans une première partie de cet exposé, nous commencerons par dégager ce qui paraît comme des constantes difficiles à dépasser dans la nature même du travail enseignant. Nous montrerons ensuite quelques inflexions et évolutions significatives comme l'évolution du travail prescrit, l'intensification et la complexification du métier, la diffraction du métier, et les sources individuelles ou collectives d'insatisfaction et de malaise parmi les enseignants. Enfin, dans une dernière partie nous nous interrogerons sur les raisons qui tendent à expliquer la relative " résistance " et froideur des enseignants, face à des réformes qui sont supposées leur vouloir du bien.

Cet exposé se fonde méthodologiquement sur une exploration de la littérature francophone mais aussi anglophone portant sur la question du travail enseignant et de son évolution. Précisons d'emblée qu'il ne s'agit pas d'un compte rendu exhaustif et complet; d'une part, il se focalise essentiellement sur les enseignants du secondaire, où les changements sociaux évoqués sont peut-être les plus prégnants, d'autre part, il est tributaire des limites de notre investigation de cette littérature assez abondante. Notre compte rendu doit être considéré comme exploratoire (3). Son ambition a été de reprendre les travaux scientifiques les plus significatifs par rapport à la question de l'évolution du travail enseignant.

\section{LES PERMANENCES DU TRAVAIL ENSEIGNANT}

Par comparaison aux travaux sur la protession et sa morphologie (Chapoulie, 1974 ; Léger, 1983 ; Berger, 1979), les études sur le travail enseignant ou sur l'organisation scolaire en rapport avec le travail enseignant n'ont pas été légion dans la littérature francophone et anglo-saxonne, selon Tardif et Lessard (1999), qui ont présenté une première synthèse importante sur ce sujet. Dans le monde anglo-saxon, ce sont surtout les travaux de Hargreaves (1994), Ozga et Lawn (1981) qui ont porté sur le travail enseignant et son contexte. Dans la littérature francophone, si certaines études du corps enseignant (Chapoulie, 1987 ; Dutercq 1993 ; Hirschhorn, 1993 ; Tanguy, 1991) ou des pratiques et compétences pédagogiques (Chapoulie, 1979 ; Perrenoud, 1993 \& 1994) abordent indirectement la question de la relation entre contexte organisationnel et travail des enseignants, les premiers travaux en France à thématiser spécifiquement l'évolution du travail et de la compétence des enseignants sont ceux de Lise Demailly (1987 \& 1991), mais aussi les travaux de E. Bautier, J.-Y. Rochex et leurs collègues (Bautier, 1995 ; Chatel et al., 1996) ceux de A. Barrère (2002), V. Lang (1999) et H. Peyronie pour les instituteurs (1998). Nous y reviendrons ci-après. Attardons nous pour l'instant au travail de Tardif et Lessard, car leur synthèse sur la question du travail enseignant permet de présenter quelques dimensions structurantes du travail enseignant, qui nous apparaissent comme des invariants du métier aussi longtemps que la forme scolaire prédominera : travail centré sur l'enseignement et les élèves, relativement structuré, protégé et isolé du travail des autres travailleurs scolaires par la forme "classe", où l'enseignant dispose d'une autonomie non négligeable, alors que par ailleurs, il est confronté, souvent seul, à une forte incertitude des situations. 


\section{Forme scolaire et travail cellulaire}

Les travaux socio-historiques de Guy Vincent (1994), ont souligné comment l'école s'est construite et développée en Europe entre le $\mathrm{XV}^{\mathrm{e}}$ et le $\mathrm{XV}\|\|^{\mathrm{e}}$ siècles en se basant sur "la forme scolaire», soit un dispositif organisationnel et institutionnel particulier qui simultanément définit une organisation spatio-temporelle (l'école comme lieu séparé, disposant d'une structure temporelle spécifique, qui l'oppose à d'autres organisations), donne forme au savoir à transmettre (un savoir structuré par la forme scripturale) et à un rapport social particulier (le rapport pédagogique de transmission enseignant-enseigné). La diffusion de cette forme explique que de nombreuses écoles présentent encore de nos jours des traits organisationnels et matériels proches des écoles du passé. L'école se développe en effet dans un espace autonome, séparé du milieu communautaire ambiant avec un rapport social spécifique de transmission qu'on appellera la pédagogie scolaire (exercices, mémorisation, répétition, examens, devoirs, récompenses et corrections, etc.), dans lequel les comportements sociaux sont fortement régulés (activités corporelles, usage et rapport au temps, usage et rapport à l'espace) et où sont utilisés divers outils relevant d'une forme scripturale (manuels, livres, cahiers, tableaux, etc.). L'enseignant y occupe un rôle et un statut central. Les écoles se ressemblent dès lors souvent en ce qu'elles reposent "sur un dispositif très simple et très stable : les classes, c'est-à-dire des espaces relativement fermés (la plupart du temps très fermés), dans lesquels les enseignants travaillent séparément en y accomplissant l'essentiel de leur tâche ». (Tardif \& Lessard, 1999, p. 56). Le travail des enseignants est donc de façon centrale « un travail cellulaire "; la division du travail repose sur une séparation des groupes d'apprentissage par matière et/ou par âge, en autant de "classes ». Dès lors, les travailleurs de l'enseignement sont séparés les uns des autres pour l'accomplissement de leur tâche d'enseignement, qu'ils accomplissent de façon " autonome, dans un lieu le plus souvent soustrait au regard des autres travailleurs ". (Ibid., p. 57). Dans cette classe, les enseignants reçoivent le double mandat de socialiser et d'instruire les élèves. Pour ce faire, il s'agira " de maintenir l'ordre dans la classe dans le but de faire apprendre les élèves; mais le maintien de l'ordre est lui-même une tâche d'apprentissage - de socialisation - tandis que l'apprentissage des connaissances scolaires est en même temps aussi apprentissage d'un certain ordre cognitif jugé légitime ". (Ibid., p. 70). Cette double mission est susceptible de dosages variés (entre les niveaux d'enseignement par exemple) et d'interprétations variées selon les contextes, notamment nationaux.

Contrairement à d'autres unités de production ou de service, cette unité de base du travail enseignant n'a pas vraiment été bouleversée depuis deux siècles. II en découle une profonde stabilité du travail de l'enseignant et le fait que leur travail est pour l'essentiel un travail individuel.

\section{Un exécutant autonome dans sa classe}

Par ailleurs, l'enseignant dispose d'une forte autonomie dans sa classe. Cette autonomie a pu être défendue par la nécessité devant lequel se trouve l'enseignant de s'ajuster à la diversité et à la complexité du travail face auquel il se trouve. En effet, "tenir sa classe " "faire apprendre les élèves " implique une activité d'une grande complexité qui varie selon l'environnement, le public élève, les buts, les moyens fixés pour les atteindre. Tardif et Lessard reprennent ainsi la description dynamique de la tâche de l'enseignant dans la classe proposé par Doyle : «il s'y produit des tâches et des évènements multiples et simultanés les- 
quels se déroulent selon une certaine immédiateté et simultanéité et une certaine rapidité ; ils comportent de l'imprévisibilité ; ils sont visibles, c'est-à-dire publics ; enfin ils se déroulent selon une trame temporelle, historique, qui renvoie à leurs conséquences sur les évènement et les tâches à venir dans la classe ». (Ibid., p. 70). Tardif et Lessard rajoutent en plus que cette multi-activité est « interactive " (les élèves et les professeurs co-présents interagissent les uns en fonction des autres) et "significative " (les interactions dépendent de la signification attribuée aux gestes et actes par les uns et les autres).

Cependant, l'enseignant n'est pas seulement un travailleur purement " autonome ». Il est simultanément un exécutant qui n'a jamais en tant que tel participé à la sélection de la culture scolaire et à la définition des savoirs prescrits dans les programmes. Il doit au minimum tenir compte de diverses injonctions et ou contraintes relatives aux objectifs, aux missions de l'école, au contenu voire à la pédagogie à utiliser, ou encore à la structure temporelle de l'enseignement. Ces derniers éléments structurent son travail même s'il lui reste encore dans les faits une large marge de manœuvre, notamment dans la sélection du savoir à transmettre au sein des programmes comme le montre Barrère (2002). Ainsi, l'enseignant a un mandat (le plus souvent double, d'instruire et de socialiser) ; son travail s'inscrit dans une organisation "disciplinaire " des programmes et un curriculum structuré selon une temporalité relativement longue. De fait, le temps scolaire va être structuré selon un découpage administratif (heures de cours, années scolaire) qui est supposé organiser l'apprentissage dans la durée (dimension historique) et renvoie à une dimension collective également dans la mesure où l'action d'apprentissage s'étale donc sur plusieurs années scolaires concernant différents enseignants.

Ainsi, le travail de l'enseignant est-il à la fois "bureaucratisé ", normé par le contexte de l'organisation scolaire et un travail « professionnalisé » qui en appelle à son initiative autonome, à ses compétences et à sa responsabilité. Cette double dimension renvoie à la double dimension de l'organisation scolaire dans son ensemble qui a été analysée par de nombreux auteurs comme une "bureaucratie professionnelle » (Bidwell, 1965 ; Maroy, 1992).

\section{Un métier relationnel et humain}

Mais cette organisation ne traite pas des objets. L'activité de base est une relation humaine, avec un enseignant qui travaille non pas «sur " des élèves humains mais " avec et pour " des élèves, surtout depuis que l'élève est mis " au centre " du système et que l'on se soucie " des besoins " de l'enfant et de la psychologie de l'enfant sous l'influence de l'Éducation nouvelle. De ce fait, l'activité est marquée non seulement par l'incertitude, mais aussi par une dimension affective, émotionnelle en plus de la dimension cognitive et intellectuelle. De plus, à la différence d'autres professions de service, cette activité présente un caractère public et visible. L'enseignant dans la classe s'adresse à un groupe d'élèves et son activité est dès lors soumise à leur regard. Sa face, son identité sont engagées dans la relation.

La nature de cette activité de service "relationnelle " et « humaine " va de pair au niveau organisationnel, avec plusieurs autres traits, comme l'ont montré plusieurs sociologues des organisations: 1) la définition des objectifs et des résultats à atteindre est incertaine, ambiguë et soumise à interprétation, de sorte qu'il sera difficile de les définir parfaitement de manière opératoire et mesurable (en particulier sur la dimension éducative) ; 2) l'incertitude concerne ensuite les 
"technologies» à utiliser: d'une part "les matériaux" sur lesquels les " méthodes ", techniques, routines du professeur s'appliquent sont "variables "; d'autre part, il n'y a pas de relations de cause à effet univoques, permettant de prédire de façon certaine les résultats de l'utilisation de telle ou telle technique. II en résulte un "flou " et une "incertitude " constitutives de ce travail (Hasenfeld \& Abbott, 1983 cité par Tardif \& Lessard, 1999; Meyer \& Scott, 1983). Dès lors, la tendance sera de confier à des « professionnels " le soin de réduire cette incertitude (4).

\section{Un métier à composante disciplinaire et intellectuelle}

Insister sur la dimension relationnelle du métier ne signifie pas que le métier d'enseignant se réduise à cette dimension psycho-sociale. La relation s'engage autour de savoirs à transmettre et à approprier. Les enseignants, au secondaire en particulier, déploient de ce fait une activité cognitive et intellectuelle importante, mobilisée lorsqu'il s'agit de sélectionner les savoirs à transmettre, de les mettre en forme pédagogique, de faire la classe, avant de contrôler l'appropriation des savoirs par les élèves. Cette dimension intellectuelle et souvent disciplinaire de l'activité des enseignants peut rester pour partie invisible, dès lors qu'elle se situe en amont, à l'aval ou à l'arrière-plan de l'acte d'enseignement dans la classe ; elle n'en est pas moins importante. Ainsi, A. Barrère (2002), à la suite d'une étude qualitative auprès d'une quarantaine d'enseignants de collège et lycée, montre qu'une des activités (subjectivement et objectivement) importantes pour les enseignants français se joue au moment des préparations, au moment de « faire le cours ». Cette activité se structure largement par rapport à une discipline, dont l'importance et la signification subjective évolue au cours de la carrière enseignante mais qui reste toujours structurante. Ainsi, préparer le cours nécessite de se situer face aux programmes scolaires. Il y a tout un travail d'interprétation, de sélection, de "bricolage " intellectuel important, qui s'applique egalement aux manuels. La construction du cours implique de plus un souci de mise en forme pédagogique des savoirs, partagé par tous les enseignants, même chez ceux qui pourraient au niveau des représentations et de l'identité, se définir comme des enseignants centrés sur "la discipline" par opposition aux "pédagogues ".

Cette dimension intellectuelle et disciplinaire du travail des enseignants, en particulier au secondaire, est fortement mise en avant dans les travaux français comme par ex. par Rochex et ses collègues (Chatel, Rochex, Roger, 1996), par Van Zanten (2001) ou encore Barrère. On le retrouve encore dans les travaux qui relèvent davantage de l'analyse du travail ou de la psychologie du travail, comme ceux initiés par les chercheurs du Laboratoire de Psychologie du Travail et de l'Action d'Y. Clot (Litim et al., 2005).

\section{Des activités diverses en relation directe ou indirecte avec les élèves}

Les tâches de l'enseignant sont difficiles à décrire in extenso car, comme on vient de le voir, le travail est simultanément prescrit par des règles administratives et bureaucratiques mais surtout modelé par l'activité responsable et autonome du travailleur. Une partie de ces tâches se déroule soit en classe à l'abri du regard d'autres professionnels, soit dans d'autres lieux que l'école (travail à domicile). Ces tâches sont dès lors difficiles à décrire et à évaluer dans leur étendue. Elles sont "extensibles" de façon plus nette que dans un travail circonscrit par une activité purement bureaucratique. Néanmoins, Tardif et Lessard montrent 
que les tâches principales des enseignants impliquent le plus souvent un rapport direct ou indirect aux élèves : outre l'enseignement proprement dit, et les tâches directement associées de préparation, de correction et d'évaluation, il s'agit de la surveillance, de la participation à des activités parascolaires, des rencontres avec les parents ou du tutorat (5). La façon dont ces tâches sont distribuées parmi les enseignants, la façon dont ils les conduisent peuvent évidemment être éminemment variables (Barrère, 2002)

\section{Une identité professionnelle plus qu'organisationnelle}

L'identité de l'enseignant est bien davantage professionnelle qu'organisationnelle, si nous entendons par ce dernier vocable, le fait que l'enseignant se définirait par rapport à l'établissement ou au système scolaire dont il fait partie. L'identité de l'enseignant est professionnelle en ce sens qu'elle se définit essentiellement en relation avec l'exercice de son métier, à partir du travail et des relations avec les élèves dans les classes, à partir du rapport aux savoirs et compétences (disciplinaires, pédagogiques, relationnels, émotionnels) que l'enseignant y engage. Cette identité est le résultat d'un processus biographique et social, qui est tributaire des contextes d'enseignement et de la biographie individuelle, mais aussi de la période historique et de l'état du système d'enseignement (Cattonar, 2002).

En effet on peut repérer historiquement différents "types identitaires " disponibles à partir desquels les identités individuelles des enseignants peuvent se construire et évoluer (Dubar, 1996, p. 112). Ainsi, V. Lang a montré que les «modèles idéaux " à partir desquels les enseignants construisent leur identité sont historiquement contingents (Lang, 1999, p. 59-107). "L'ethos des enseignants » au début $d u x x^{\mathrm{e}}$ siècle se fondent sur l'attachement à une compétence culturelle, à la maîtrise d'une discipline et les savoir-faire pédagogiques sont secondaires. I e modèle de professionnalité de référence relève dı modèle dı "magister" (Ibid., p. 96). Il cite plusieurs enquêtes contemporaines qui confirment le poids encore actuel de cette tradition; cependant, des évolutions sont en cours, notamment l'émergence de la figure du "pédagogue » (Ibid., p. 100), qui s'accompagne d'un déplacement de l'attention portée sur la connaissance à transmettre vers la connaissance à apprendre, voire l'apprentissage de la connaissance (Ibid., p. 103). Cette évolution du modèle du «magister » (enseignants centrés sur les savoirs à transmettre) à celui du "pédagogue " (enseignants centrés sur le processus d'apprentissage et les élèves) est également constatée chez les enseignants par J.-F. Blin (1997), et par F. Dubet qui observe un déplacement de l'identification disciplinaire vers "l'art " d'enseigner, du goût pour la discipline enseignée vers l'intérêt pour l'enseignement, "faire cours" (Dubet, 1991, p. 302). Ces modèles de référence se distribuent de plus inégalement selon les degrés d'enseignement, dans la mesure où le modèle du magister est plus prégnant parmi les enseignants du second degré que chez les instituteurs du primaire. (Dubet, 2002). Par rapport à ces définitions emblématiques, Barrère (2002) souligne cependant que les enseignants qu'elle a étudié dans son étude qualitative «font de la pédagogie, mais ne se définissent que rarement comme pédagogues " (p. 84) ; la pédagogie ne "se lit pas", elle est un savoir pratique difficile à formaliser mais, elle est présente chez tous comme une préoccupation et une réflexion pratique de l'enseignant, attentif à ce que le cours préparé "fonctionne " ensuite comme cours fait.

Cattonar et Maroy (1999) à partir d'une analyse de discours des principaux acteurs non-enseignants (6) intervenant dans le champ éducatif en Belgique 
francophone révèlent une même tendance et évolution du modèle « idéal " de l'enseignant. Ils ont ainsi observé une remarquable convergence dans la définition de ce que devrait devenir aujourd'hui l'enseignant au regard des évolutions de l'école et de la société. Un «nouveau modèle de professionnalité enseignante $(7)$ : celui du "praticien réflexif " (proche du modèle du "pédagogue " décrit par Lang et Blin) est promu au détriment de modèles anciennement valorisés comme celui du "maître instruit " (qui maîtrise avant tout les savoirs disciplinaires, travaille individuellement, mène une pédagogie de type "monolithique " et « transmissive », en se référant aux prescriptions formelles provenant des autorités scolaires) ou celui de "l'enseignant technicien " (qui possède et applique un répertoire de techniques pédagogiques et savoir-faire procéduraux précis et efficaces, dérivés d'études scientifiques). L'enseignant «idéal » est perçu aujourd'hui par les différents acteurs éducatifs comme devant être un "praticien réflexif ", qui mène une pédagogie de type constructiviste et différenciée, qui travaille en équipe et s'investit dans la gestion collective de la vie de son établissement. Ce modèle diffusé parmi les acteurs décideurs a été largement promu et développé par des pédagogues dans plusieurs pays francophones (Perrenoud, 1993 ; Paquay, 1994 ; Paquay et al. 1996), en s'inspirant au départ des travaux de Schön (1983).

Par ailleurs, Lessard et Tardif (2004) notent également que le rapport au travail enseignant a évolué passant d'un travail envisagé comme "vocation " (insistant dès lors à la fois sur les qualités morales et le savoir disciplinaire), au " métier " (impliquant des savoirs techniques) et enfin à la " profession " reposant sur une expertise et capacité de jugement réflexif de haut niveau.

Par delà les variations historiques, des variations dans l'espace existent aussi. Mac Ness et al. (2003) suggèrent que des variations nationales existent entre les différentes conceptions du travail enseignant (au secondaire) telles qu'ils apparaissent au travers des missions prescrites aux enseignants. Ainsi c'est en $\Gamma$ rance que la définition des missions de l'enseignant secondaire sont le plus nettement énoncés en termes académiques, laissant le côté éducatif, affectif à une profession complémentaire, les " conseillers principaux d'éducation ". À l'inverse, au Danemark ces deux volets sont étroitement liés par rapport au même groupe classe, alors qu'en Angleterre, l'enseignant est tenu de jouer ces deux rôles, mais par rapport à des groupes d'élèves différents.

\section{LES CHANGEMENTS DU TRAVAIL ENSEIGNANT : UNE DIVERSIFICATION DES TÂCHES PRESCRITES}

Si plusieurs traits clés du métier enseignant constituent des dimensions relativement stables dans le temps et partiellement dans l'espace, la littérature actuelle sur le métier enseignant insiste aussi sur les inflexions et les changements qui seraient en cours d'émergence, soit sous l'effet de transformations générales de la société et de l'institution scolaire, soit en raison de politiques éducatives. Nous venons de pointer les évolutions des modèles de professionnalité et types identitaires de référence. Nous allons aborder à présent les évolutions effectives du travail enseignant en reprenant la distinction des sociologues du travail entre le travail prescrit et le travail réel.

Nous partirons tout d'abord des évolutions souhaitées, voulues, encouragées, prescrites par les autorités scolaires. En effet, les politiques de modernisa- 
tion de l'école touchent tant la définition de l'établissement que la définition de la " professionnalité enseignante " (Maroy, 2002d). Le modèle de l'établissement qui s'impose est celui de "l'établissement mobilisé " par un projet éducatif, dynamisé par un directeur qui n'est plus seulement un primum inter pares mais un véritable "manager scolaire", autour d'une équipe enseignante, innovante et coopérative qui cherche à améliorer ses pratiques et à évaluer son action (Dupriez, 2002 ; van Zanten et al., 2002) (8). Parallèlement, la professionnalité enseignante est redéfinie en insistant sur ses compétences pédagogiques, sa réflexivité, sa capacité de développement professionnel ou encore sa capacité à travailler en équipe (Paquay et al., 1996). Les transformations des compétences et pratiques formellement attendues des enseignants, soit le travail prescrit aux enseignants, est en effet en train d'évoluer dans plusieurs pays européens et c'est en soi un changement significatif, même si le travail réel n'évolue pas forcément au même rythme ni dans la même direction.

Ces changements des prescriptions à l'égard du travail enseignant avait déjà été identifiée dans les années 1980 par Lise Demailly (1987) qui étudiait les pratiques professionnelles des enseignants et les processus de redéfinition de leur compétence professionnelle en France. Sous l'influence de divers facteurs (évoIution du public scolaire, directives ministérielles) et non sans médiation par des luttes autour de l'enjeu de la compétence, elle montre que les savoirs et aptitudes pratiquement requises par les situations professionnelles des enseignants évoluent : la part de la qualification (fondée sur des titres scolaires essentiellement) est globalement minorisée au profit de nouvelles capacités professionnelles, non réglementairement exigibles, mais socialement et institutionnellement valorisées, au sein desquelles elle distingue une composante éthique et une composante organisationnelle. À l'époque, elle montre que ces nouvelles composantes ne sont pas encore réglementairement inscrites dans une redéfinition formelle des compétences attendues, mais qu'on assiste à une redéfinition de la pratique enseignante légitime, supportée par de nouveaux modes de contrôle (locaux, diffus, de nature plus pédagogique) et de nouveaux styles de formation continue.

Depuis lors, ces changements de la professionnalité attendue des enseignants peuvent se repérer dans des changements plus formels (de nature législative et réglementaire) opérés dans plusieurs pays européens. On peut en trouver des indices à partir des données institutionnelles produites par Eurydice et I'OCDE ces dernières années concernant la profession enseignante (Eurydice, 2001,2002 \& 2003 ; OCDE, 2005). Ces travaux renseignent sur les changements législatifs et réglementaires opérés dans divers systèmes scolaires européens et dans le même temps, ils les encouragent.

En effet, ces travaux renforcent d'abord le changement de référentiels normatifs en précisant ce que dorénavant l'enseignant doit être ou devenir. Par exemple Eurydice rappelle qu'une des deux grandes problématiques qui émerge des débats politiques dans la plupart des pays européens est celle de « la modification du profil de compétences attendu des enseignants ", l'autre étant celle de "l'attractivité du métier ". Ainsi, " dans la majorité des pays, on n'attend plus uniquement des enseignants qu'ils s'adaptent, dans leur mission actuelle d'enseignement, aux connaissances que nous procure la recherche sur la didactique et sur la psychologie de l'apprentissage. Le développement technologique de l'information, une vie sociale toujours plus multiculturelle, l'autonomie croissante accordée aux communautés locales et aux établissements scolaires, etc. influencent la vie de l'école. De manière générale, la profession enseignante serait donc concernée par la nécessité de s'impliquer davantage dans des tâches administratives et 
de gestion scolaire, d'utiliser les technologies de l'information et de la communication, de promouvoir les droits humains et l'éducation civique, et de former les élèves à apprendre dans une perspective d'apprentissage tout au long de la vie. Dans ce contexte, les enseignants doivent aussi faire face à des groupes d'étudiants qui n'ont jamais été aussi hétérogènes " (Eurydice, 2003, p. X).

Ensuite, ces travaux de comparaison entre système européens montrent que, d'ores et déjà dans plusieurs pays, les politiques éducatives ont conduit à une redéfinition des tâches requises, que le mode de définition du temps de travail prescrit est aussi en train d'évoluer, et que finalement, le niveau et l'acteur compétent en matière de "gestion " du temps de travail de l'enseignant est de plus en plus l'établissement et son directeur.

Ainsi, les travaux d'Eurydice (Eurydice, 2003 p. 33-40) soulignent que « traditionnellement, en Europe, le temps de travail des enseignants était établi en nombre d'heures d'enseignement. Cela correspondait au profil des tâches de l'enseignant qui se définissaient en deux activités principales: les cours d'une part, les préparations/corrections d'autre part. ". Seuls quatre pays sur les trente analysés (9), ont conservé ce «mode traditionnel " d'évaluation du temps prescrit (Belgique, Allemagne, Irlande et Luxembourg).

Or dans d'autres pays, pour mieux prendre en compte, stimuler et réguler les autres activités des enseignants, le mode de définition du temps de travail a été modifié. Ainsi dans quatorze pays, "la définition a évolué avec l'ajout, au volume d'heures d'enseignement, d'un nombre précis d'heures ou de jours de présence à l'école pour d'autres activités spécifiques telles que le travail d'équipe, des tâches de gestion, etc. La majorité de ces pays donnent également une indication du temps de travail global. » (Ibid., p. 34). Trois pays enfin proposent une définition du temps de travail des enseignants sans plus aucune référence précise au nombre d'heures d'enseignement à donner, soit qu'ils définissent un temps de travail global annuel (Pays-Bas et Suède), soit un "temps dirigé " c'està-dire le nombre d'heures où l'enseignant doit être disponible pour des prestations à l'école sous la responsabilité du chef d'établissement (Angleterre, Paysde-Galles). Le reste (neuf pays) précise un nombre d'heures d'enseignement et un temps global de référence à prester (dont la France).

Eurydice, qui se réjouit de cette évolution, souligne par ailleurs les enjeux d'une telle redéfinition du temps de travail enseignant: outre la reconnaissance par la société de l'ampleur de la charge de travail des enseignants, deux autres enjeux (le contrôle et la régulation des activités des enseignants; le développement d'une "latitude des écoles dans le management de leurs ressources humaines") montrent bien le lien qu'il y a entre cette redéfinition du travail prescrit et le développement d'une logique d'un « établissement plus autonome " qui devrait conduire à une amélioration de qualité et d'efficacité de l'enseignement.

Une autre comparaison faite par Eurydice concerne les tâches statutairement requises des enseignants. Bien qu'ils reconnaissent que ces tâches prescrites peuvent considérablement s'écarter du travail réel, ils s'interrogent sur le fait qu'au-delà de l'enseignement proprement dit (et des tâches directement liées, préparation, correction et évaluation), d'autres tâches soient prescrites pour l'enseignant individuellement (supervision d'élèves pendant ou après les cours, remplacement) ou pour les enseignants en équipe. Ils concluent que "dans tous les pays, selon la loi ou sur la base des conventions collectives, les enseignants sont supposés mener un travail en équipe, c'est-à-dire se coordonner entre eux et collaborer activement dans différents domaines (évaluation interne de l'école, pré- 
paration du programme, plan d'activité de l'école, etc.). Ce travail en équipe n'engendre pas de rémunération supplémentaire ni de diminution du temps d'enseignement. " (Eurydice, 2003b, p. 2)

On le voit ces évolutions du travail prescrit, encouragées par nombre de travaux de pédagogues qui ont travaillé à la modélisation de nouveaux modèles de professionnalité commencent à trouver dans les législations quelques concrétisations effectives. Ce constat est renforcé par ce qui se joue en ce qui concerne la nécessité du développement professionnel et de la formation «permanente » en cours de carrière. Ainsi d'après Eurydice (2003b, p. 3), " les enseignants de la moitié des pays européens sont soumis à l'obligation professionnelle d'actualiser leurs connaissances. Dans six autres pays, la formation continue peut être considérée de facto obligatoire étant donné qu'aucune promotion ne peut être accordée aux enseignants qui n'ont pas participé à ce type d'activités. ". L'offre de formation est de plus très rarement aux mains du ministère central seul (quatre petits pays). Elle est le plus souvent partagée entre pouvoirs régionaux et locaux, voire totalement décentralisée au niveau des établissements (sept pays)

Dans les travaux de l'OCDE (2005, p. 86-87), consacré aux stratégies à mener face aux pénuries des enseignants, on trouve également une analyse de l'évolution des tâches que l'enseignant est susceptible de pouvoir assumer. La liste large établie par l'OCDE recoupe assez largement plusieurs dimensions du modèle du praticien réflexif déjà évoqué (insistance sur le fait d'être capable de stimuler et gérer les processus d'apprentissage des élèves, capacités de faire face à des classes hétérogènes, à travailler en équipe, implication dans l'organisation de l'école par exemple).

\section{TRAVAIL RÉEL ET CONDITIONS DE TRAVAIL : INFLEXIONS ET DIFFRACTION}

Si le travail prescrit évolue dans le sens d'une diversification, qu'en est-il du point de vue du travail réel ? Que sait-on de son évolution ? De l'évolution des conditions de travail de l'enseignant? Comment ces évolutions se traduisentelles dans les subjectivités enseignantes, dans leur rapport au travail, leurs insatisfactions ou satisfactions professionnelles?

\section{Intensification, diversification et complexification du travail}

Tant les travaux anglo-saxons que francophones s'accordent pour avancer que dans les faits, le métier enseignant devient plus complexe et plus diversifié et que dès lors on assiste à une forme d'intensification de la charge de travail des enseignants. Cette intensification du travail serait moins liée à un allongement du temps de travail des enseignants proprement dit qu'à la complexification du métier. Dans la littérature francophone, cet état des choses est surtout rapporté à l'évolution du public scolaire. La difficulté de réaliser les missions de l'école dans une société en mutation est également mise en avant. Dans la littérature anglo-saxonne, on relève de surcroît des difficultés liées aux conséquences de la mise en œuvre des politiques éducatives, qui engendrent par elles-mêmes à la fois une tendance à la surcharge de travail et à des tensions accrues dans l'exercice du métier.

\section{Le temps de travail}

Le temps de travail des enseignants est difficile à estimer et à comparer entre les différents pays européens, en raison d'un décalage entre le temps de travail 
statutairement prescrit et le temps de travail réel, qui déborde les seules heures d'enseignement ou de présence obligatoire à l'école et incorpore les préparations, corrections à domicile, etc. Selon les méthodes utilisées pour cerner le temps de travail « non statutaire ", les données varient.

Au niveau des comparaisons internationales, Eurydice (2003) s'est borné à comparer les temps de travail officiellement prescrit et constate de ce point de vue une assez grande variété qui nous renseigne peu sur les heures prestées effectivement. Cependant, sans véritablement documenter l'affirmation, le rapport indique que la charge de travail prescrite serait en augmentation constante dans de nombreux pays notamment pour ce qui concerne les pays suivants: Belgique - Communauté française, Danemark, Grèce, Espagne, France, Finlande, Suède, Royaume-Uni, Islande, Lettonie, Pologne, Roumanie, Slovaquie (Ibid., p. 44).

Tardif et Lessard (1999) proposent aussi une comparaison des temps de travail statutaires dans les pays de l'OCDE, à partir des heures d'enseignement officiellement prescrites, la moyenne s'établissant à 23,1 heures d'enseignement par semaine pour l'enseignement primaire et à 19,3 heures pour l'enseignement secondaire (p. 122-123).

Des enquêtes nationales existent, basées sur des méthodologies variées (enquêtes ou semainiers) : ainsi par exemple Chenu (2002), à partir des enquêtes "Emploi du temps » de l'INSEE (1998-1999) établit que pour la France les enseignants (instituteurs, et professeurs) travaillent 36 heures en moyenne et connaissent un temps de travail "dual " qui est composé essentiellement de "deux composantes nettement disjointes, les heures statutaires d'enseignement dont le rythme, en grande part, s'impose à l'enseignant (y compris assez souvent le samedi matin), et le temps de préparation et de corrections, réparti de manière bien plus libre " (p. 160). Par ailleurs, ils connaissent une moindre proportion de "semaines normales de travail " que les autres catégories de travailleurs, lesquels travaillent en moyenne plus longtemps.

Ces chiffres apparaissent en retrait par rapport à d'autres estimations. Un des raisons de cet écart est méthodologique et liée au mode de questionnement. Le minimum d'heures est atteint dans les enquêtes emploi où beaucoup d'enseignants donnent une estimation qui n'inclut que leur travail statutaire, alors que les enquêtes spécifiques au Ministère de l'éducation aboutissent à des estimations plus élevées car leur questionnaire évoque au titre du temps de travail, un temps « consacré à des recherches personnelles".

En fonction des options méthodologiques prises par les différentes enquêtes menées en France, on constate ainsi des estimations très variées du temps de travail des enseignants. Barrère (2002) synthétise ces résultats variés de la façon suivante : "Claude Thélot indique une fourchette hebdomadaire comprise entre 29 heures et 36 heures de travail, l'horaire statutaire devant les élèves étant de 15 heures pour un professeur agrégé et de 18 heures pour un certifié. D'autres enquêtes effectuées par la Direction de l'évaluation et de la prospective arrivent au total de 39 heures avec des variations suivant l'établissement et la matière. Mais bien d'autres enquêtes dépassent ce chiffre pour aller jusqu'à des totaux de 42 heures en moyenne; une étude du Syndicat national des enseignements de second degré (SNES-FSU), réalisée au printemps 1998, arrive à une moyenne de 44,8 heures. " (Barrère, 2002, p. 16-17 ; voir aussi France, 1996 ; Baumard, 2004)

En Communauté française de Belgique, Maroy (2002) établit un temps de travail de 36 heures à partir d'une enquête auprès des enseignants du secondaire. Par contre, Alt et al. (1999) estime le temps de travail des enseignants aux États- 
Unis à 45 heures en 1993-1994, un temps de travail comparable à ce que prestent d'autres professionnels selon cette enquête.

Par ailleurs, peu d'enquêtes ont établi si on assistait à une augmentation ou une diminution du temps de travail des enseignants. On peut seulement avancer que selon Eurydice (2003), le temps d'enseignement prescrit des enseignants n'a pas diminué. Par ailleurs, des tâches nouvelles leur ont été attribuées (mais ne sont pas pour autant toujours effectives), sans que ce ne soit compensé ni par une augmentation salariale, ni par une diminution de l'horaire dans d'autres domaines. Par ailleurs, cette stabilité du temps de travail prescrit ou statutaire contraste avec une tendance à la diminution du temps de travail prescrit dans d'autres secteurs publics. Au minimum, on peut dès lors sans doute avancer que le temps de travail effectif des enseignants n'a pas diminué, contrairement à d'autres catégories de personnel du secteur public.

Cette hypothèse d'une stabilité du temps de travail enseignant est aussi avancée par Tardif et Lessard (1999) qui, estiment, à partir de plusieurs enquêtes québécoises, que "la semaine normale de travail de la vaste majorité des enseignants tourne autour de 37 à 40 heures, soit 27 heures obligatoires de présence à l'école, 5 à 8 heures à la maison, le reste du temps occupé à d'autres activités à l'école ou ailleurs (activités parascolaires, formation, etc.). Ces informations laissent à penser que la charge de travail, évalué en nombre d'heures par semaine, n'a pas vraiment changé depuis une trentaine d'années. Comme nous le verrons plus loin, s'il y a un accroissement de cette charge, celui-ci est d'abord qualitatif. » (Tardif \& Lessard, 1999, p. 142). Plutôt que dans l'allongement du temps de travail, l'intensification de leur travail se manifeste davantage par la multiplication et la complexification des tâches des enseignants.

\section{La gestion de la classe de plus en plus complexe et difficile}

Tout d'abord, au cœur du métier soit dans le travail d'enseignement dans la classe, la gestion de la classe devient plus difficile et implique des compétences très diverses (Mac Ness et al., 2003 ; Rayou \& van Zanten, 2004 ; Tardif \& Lessard, 1999 ; Chatel et al., 1995). En effet, les « routines traditionnellement diffusées par l'institution ne permettent plus de faire face aux situations. Le travail des enseignants devient tout autant émotionnel qu'intellectuel, car il faut mobiliser, outre les savoirs académiques, des connaissances et savoir-faire divers pour assurer des interactions qui rendent possible l'apprentissage. " (Rayou \& van Zanten, 2004, p. 31). Cette situation est liée à plusieurs évolutions clés du système scolaire.

Première évolution majeure : avec l'extension de la scolarité et la promotion d'un tronc commun dans les premières années du secondaire (collège unique, comprehensive school), le public scolaire est devenu plus hétérogène et plus "difficile» (Dubet, 1991; Lessard \& Tardif, 1996; Perrenoud, 1993). Avec la "massification" du système scolaire, les conditions sociales et culturelles qui favorisaient auparavant la participation et l'écoute des élèves et encourageaient un rapport positif à l'apprentissage et aux savoirs ne sont plus données a priori par le contexte familial et social des élèves. De ce fait, on se retrouve avec multiples élèves qui ne " maîtrisent " plus le métier d'élève et les enseignants doivent prendre en compte différentes logiques et rapports au savoir et à l'école : logique de cheminement et de survie dans le système ou logique d'apprentissage et de développement (Bautier \& Rochex, 1997). Ce phénomène est accentué par l'affirmation d'une culture juvénile qui tend également à mettre à distance les valeurs 
et les pratiques scolaires. (Barrère, 2002 ; Dubet \& Martucelli, 1996 ; Rayou \& van Zanten, 2004).

Les savoirs enseignés ont en outre connu une certaine inflation et un renouvellement rapide engendrant chez les enseignants une relative incertitude sur ce qui vaut d'être enseigné (Perrenoud, 1993). Enfin, l'arrivée de nouveaux "spécialistes de la jeunesse » (éducateurs, animateurs, etc.) et des nouvelles technologies (médias audiovisuels, télématique, etc.) brise le "monopole " que les enseignants détenaient en matière d'éducation et d'instruction et les oblige à redéfinir les limites de leur " compétence professionnelle» (Duru-Bellat \& Henriot-van Zanten, 1992).

Ensuite, les besoins de la société et ses attentes vis-à-vis de l'école se sont complexifiés et les finalités éducatives se sont multipliées (comme l'instruction, l'éducation, l'épanouissement du jeune, la citoyenneté, la préparation au marché de l'emploi, etc.). Par ailleurs, les enseignants sont aujourd'hui soumis à de multiples exigences et demandes éducatives, qui renvoient parfois à des logiques en tension (comme la sélection et l'égale réussite de tous) en particulier dans le premier cycle du secondaire ou au collège.

Dès lors, l'instabilité des situations scolaires dans les classes s'accentue et le travail d'enrôlement des élèves pour les inscrire dans leur métier d'élève est très important. D'où des enseignants " mis à l'épreuve " subjectivement dans la relation pédagogique quotidienne, dans la gestion de la classe. Ils doivent mettre en œuvre de façon plus cruciale de nouvelles compétences relationnelles et émotionnelles, sans doute davantage qu'auparavant. Barrère (2002) montre ainsi par une étude qualitative à quel point les compétences relationnelles et émotionnelles sont décisives pour gérer sa face et l'interaction tant dans des contextes d'enseignement "favorisés " que dans des collèges "difficiles". Le contrôle de soi et de ses affects, la gestion de la distance avec les élèves, la gestion des stigmates des élèves confrontés à des verdicts scolaires négatifs sont ainsi cruciaux.

Mac Ness et al. (2004) insistent aussi sur le fait que l'activité d'enseignement est une « activité complexe avec plusieurs dimensions contradictoires : la maîtrise de la matière, des compétences organisationnelles et pédagogiques pour planifier et évaluer l'apprentissage, des aspects sociaux et affectifs liés à l'investissement de soi » (p. 244 ; nous traduisons). Le métier demande une implication personnelle et morale importante, "un sens des responsabilités et une conscience morale ". De ce fait, les compétences à mobiliser sont multiples : " ce que signifie d'être un "bon" professeur n'est dès lors pas seulement un composé de connaissances et d'habiletés professionnelles, mais aussi une capacité d'empathie et de relation " ( $(\mathrm{bid}$.), quel que soit d'ailleurs le niveau de l'enseignement, primaire ou secondaire.

Chatel et al. (1996) montrent aussi que, dans le contexte susmentionné, la compétence et la professionnalité enseignante sont incitées à se modifier car on ne peut plus « tenir pour acquise la capacité des élèves à donner sens aux apprentissages qu'on leur propose [...] Le rapport de sens, entre apprenants et contenus ou activités d'apprentissage demande, beaucoup plus souvent qu'auparavant, à être construit, et ce d'autant plus que la pression du chômage tend à rabattre la question du sens des apprentissages et de l'expérience scolaire sur celle de leur utilité » (p. 332-333). Dès lors, outre des composantes éthique et organisationnelle déjà mises en avant par L. Demailly, les compétences professionnelles des enseignants français tendent à intégrer au-delà des contenus disciplinaires, des préoccupations relatives à « la mobilisation des élèves et leurs apprentissages ». Cepen- 
dant plusieurs logiques coexistent à cet égard dans les pratiques enseignantes, logiques contrastées selon leur plus ou moins grande distance aux enjeux disciplinaires et épistémologiques de l'enseignement: 1) réinterroger la nature des contenus et activités d'enseignement et faire un investissement didactique pour rendre les savoirs scolaires appropriables, une fois dépouillés de ce qui peut les "obscurcir» ; 2) adapter contenus et situations d'apprentissage aux contextes dont les élèves sont familiers ; 3 ) se focaliser sur ce qui parait le plus utile du point de vue des élèves, dans leur vie quotidienne immédiate ou dans leur futur professionnel.

Casalfiore \& De Ketele (2002) montrent enfin que les enseignants secondaires belges développent trois types d'activité en classe. Outre les activités d'enseignement et de transmission de la matière ("gestion de la matière "), les activités de "gestion de l'ordre " en classe classiquement distingués par la littérature, des activités de "gestion de l'engagement » visant à créer la motivation à apprendre, à intéresser et enrôler les élèves dans leur métier d'élèves sont aussi clairement identifiables.

\section{Une diversification des rôles et des tâches dans l'établissement}

Au-delà de la gestion de la classe, une plus grande variété de rôles et de tâches sont demandées à l'enseignant. Nous avions déjà repéré cette diversification au niveau des tâches prescrites. Elle semble exister au niveau des tâches réelles. Ainsi nombre d'auteurs reconnaissent que le nombre de tâches et de rôles différents à assumer dans l'exercice du métier, au moins au niveau secondaire, est plus diversifié, car de nouveaux rôles de "travailleur social, d'éducateur et de psychologue " (Rayou \& van Zanten, 2004, p. 30) s'imposent. Ces rôles sont d'ailleurs plus ou moins acceptés comme partie intégrante de la tâche (Cattonar, 2002). Néanmoins, cette situation peut être très variable selon les types d'établissements où se trouvent l'enseignant (van Zanten, 2001) dont le public scolaire et les problèmes de "maintien de la paix scolaire " peuvent être très différents en raison de phénomènes de ségrégation scolaire.

L'élargissement des rôles ne se fait pas seulement en relation avec des "métiers du social " mais d'autres rôles plus "gestionnaires " sont également évoqués. Ainsi Eurydice (2003) attribue l'augmentation de charge de travail statutaire des enseignants à l'augmentation des tâches de gestion (notamment pour la Grèce, la Suède, l'Angleterre et le pays de Galles), à l'introduction d'activités de coordination et de travail en équipe (Espagne, Suède et Pologne). C'est dire donc que l'intensification procède aussi des politiques menées dans les différents systèmes scolaires.

Des études anglo-saxonnes montrent que cette intensification du travail ne se limite pas à l'Europe et qu'on en trouve des traces tant au Canada qu'en Australie. De plus, elles soulignent qu'elle peut paradoxalement trouver sa source dans l'implication professionnelle et l'ethos professionnel des enseignants euxmêmes.

Ainsi Hargreaves (1994, p. 108, notre traduction) décrit l'intensification du travail enseignant comme « l'escalade de pressions, attentes, contrôles concernant ce que les enseignants font ou devraient faire dans leur journée de travail ". Les principaux corollaires de cette intensification sont les suivants (Ibid., p. 118-120) : 1) un manque de temps avec peu de temps pour la détente ou le développement des compétences ; 2) une surcharge chronique ; 3) le remplacement du temps passé dans la relation aux élèves par la réponse aux demandes administratives ; 4) la diversification de leur expertise ; 5) un curriculum et une pédagogie davan- 
tage cadrés et formatés (packaged). Hargreaves montre à partir d'une enquête qualitative auprès d'enseignants de la région de Toronto, que la charge de travail supplémentaire est renforcée par l'implication et l'engagement autonome des enseignants liés à leurs "ethos professionnels ". Autrement dit, les enseignants tendent à surtravailler pour simultanément répondre aux exigences externes et réaliser au moins partiellement leurs conceptions propres du métier.

Easthope et al. (2000) font un constat proche à partir d'une étude qualitative australienne: l'intensification ne dérive pas seulement des prescriptions ministérielles, mais aussi du souci des enseignants de rendre compatible leur "propre éthos professionnel " et les demandes de l'administration exigeant plus de travail et l'exercice de responsabilités plus variées: plus de travail social, plus de travail administratif, plus d'implication dans de multiples innovations relatives au curriculum, à l'évaluation.

Cette combinaison d'élargissement et de complexification du métier semble au cœur de l'alourdissement de la charge de travail des enseignants.

\section{DEUX MÉTIERS D'ENSEIGNANT ?}

Les phénomènes de ségrégation scolaire qui peuvent présenter des ampleurs variables selon les pays (Gorard \& Smith, 2004 ; Dupriez \& Vandenberghe, 2004) conduisent à une différenciation des établissements du point de vue des problèmes d'ordre scolaire ou d'apprentissage auxquels ils sont confrontés. Cette différenciation entre "établissements difficiles " et "établissements favorisés " ont amené plusieurs auteurs à avancer l'hypothèse qu'on assisterait à une différenciation des conditions d'exercice du métier d'enseignant, voire à l'apparition de métiers pratiquement différents.

Les conditions d'enseignement dans les établissements "difficiles" et les stratégies qu'elles suscitent de la part des enseignants ont ainsi fait l'objet des travaux de Jean-Yves Rochex (1995) ou d'Agnès van Zanten (2001) travaillant sur les collèges en "banlieue ". A. van Zanten montre ainsi une diversité de réponses et de formes d'adaptation des enseignants. D'une part, "travailler en banlieue" peut susciter des "stratégies de fuite " qui peuvent prendre la forme de l'abandon de la profession, la recherche d'une mobilité horizontale vers d'autres établissements ou encore le retrait intérieur, compensé par des satisfactions extrinsèques au travail d'enseignement stricto sensu. D'autre part, elle observe des "stratégies de survie " mises en œuvre par les enseignants pour préserver leur santé physique et mentale, mais aussi leur estime de soi et leur capacité à poursuivre leur projet professionnel. Les enseignants expérimentent et font ainsi place de façon variable à diverses tactiques orientées vers la " domination ", la " négociation » ou encore le «marchandage » avec les élèves. À plus long terme, ils s'adaptent à ce contexte de façon plus profonde par d'importantes « réorganisations subjectives, des bifurcations symboliques " (p. 220) qui ne relèvent pas de la " simple réaction à des situations ". L'adaptation s'opère dans le domaine du maintien de l'ordre tantôt par des stratégies plutôt masculines de domination directe tantôt par des stratégies de contrôle des classes par des techniques plus "douces» (connivence, humour, présence, capacité d'anticipation, réputation). Mais l'adaptation contextuelle s'opère aussi dans le domaine de la transmission des connaissances. Un travail de " deuil " des modèles et projets pédagogiques forgés en formation initiale ou dans d'autres contextes d'enseignement s'opère 
ainsi. Tiraillés au départ entre le souci de répondre aux exigences des instructions officielles et la nécessité de s'adapter aux situations d'enseignement, ils s'adaptent progressivement au public en acceptant de sélectionner assez radicalement les éléments à enseigner à l'intérieur des programmes et d'adapter les objectifs au niveau supposé des élèves. Ils s'adaptent aussi par la place accordée au travail de "préparation des élèves au travail ", par diverses stratégies didactiques (travail de simplification, recours à l'oral et à l'image) ou pédagogiques (jeu, travail en groupe, etc).

À partir d'une étude qualitative auprès d'une quarantaine d'enseignants répartis tant dans des collèges que des lycées scolairement et socialement diversifiés, Barrère (2002, p. 237-285) documente et argumente également qu'il y a une spécificité dans le travail enseignant au sein des établissements " difficiles", même si il ne faut pas durcir le tableau et sous-estimer les situations difficiles dans les bons établissements. Cette spécificité est ainsi davantage une différence de degré qu'une différence de nature du travail enseignant, qui réside non pas "dans la somme de travail " mais dans le « recentrage de l'ensemble des tâches autour de la gestion de la classe en tant que telle, et d'une intensification du travail relationnel avec les élèves. C'est aussi dans la nature des épreuves subjectives qu'ils traversent que vont se différencier, selon les contextes et selon les personnes, les expériences professionnelles. " (Barrère, 2002, p. 238).

Ainsi, plusieurs traits semblent plus spécifiques au travail dans les contextes difficiles : un travail collectif en établissement plus intense; une préparation de cours moins lourde et en tous cas moins orientée vers le contenu à transmettre que dans les autres établissements; mais surtout une centration de l'énergie de l'enseignant sur la gestion de la classe et de son imprévisibilité (gestion " d'incidents " relativement variés en relation avec le "verdict scolaire ", l'exercice de l'autorité, les tensions et violences entre élèves, ou la personne même de l'enseignant). Face à ces incidents, l'engagement personnel et moral de l'enseignant est crucial et Barrère décrit multiples compétences socio-affectives déployées par l'enseignant en situation pour y faire face. Cattonar (2005) suggère un diagnostic proche à partir d'une enquête qualitative et quantitative réalisée auprès d'enseignants de trois écoles secondaire belges dont le public et les filières scolaires sont socialement et scolairement très contrastés.

Cette différenciation des conditions d'exercice des enseignants explique d'ailleurs pour partie les variations dans la satisfaction professionnelle des enseignants. Bien plus que les conditions matérielles, ce sont les conditions relationnelles de travail qui affectent la satisfaction au travail. $\grave{A}$ partir d'une analyse multivariée réalisée sur les données de l'enquête du GIRSEF auprès de 3600 enseignants du secondaire belges, Maroy montre que la difficulté ressentie du travail en classe (qui est corrélée aux caractéristiques objectives des établissements d'enseignement) est un des meilleurs prédicteurs de l'insatisfaction professionnelle (Maroy, 2002). Par ailleurs, le climat des relations au directeurs ou aux collègues importe également davantage que les conditions matérielles de travail, comme la qualité des locaux ou des équipements. Les variables individuelles jouent peu (origine sociale, diplôme, âge) mis à part le genre (les hommes étant plus insatisfaits).

Toutes ces transformations ont non seulement modifié les conditions de l'exercice professionnel et rendu plus complexe et incertaine la tâche des enseignants (Delvaux et al., 1996), mais elles semblent aussi remettre en cause leur identité professionnelle (Lang, 1999). Le cahier des charges prescrit à l'enseignant s'avère parfois impossible à réaliser entièrement et peut dès lors conduire 
les enseignants à une "souffrance subjective profonde " (Cornet, 1999). Remplir différents rôles à la fois peut s'avérer épuisant et rendre plus difficile la construction d'une identité professionnelle forte (Tardif \& Lessard, 1999). Différents auteurs mettent dès lors en avant la " désinstitutionalisation de l'école " et celle, concomitante, du rôle des enseignants (Dubet \& Martucelli, 1996 ; Dubet, 2002). Cousin (1998) relève ainsi que les enseignants ont le sentiment de vivre une "perte de sens" de leur métier et il constate que les conflits au sein des écoles se cristallisent souvent autour de la définition du métier : les enseignants n'ont plus de points de repère clairs, ne savent plus sur quoi s'appuyer et les établissements apparaissent souvent comme une "succession d'individualités et de personnalités ". Demailly (1991) parle également " d'effondrement des systèmes symboliques " partagés par les enseignants et de « désenchantement des mythes qui sont le support de la vocation ".

Ce malaise et ces insatisfactions encouragent dès lors diverses stratégies visant à échapper à ces situations : depuis la " carrière horizontale " en France, en passant des souhaits de fuite du métier d'enseignant qui ne peuvent pas toujours se concrétiser. Ainsi, dans l'enquête du GIRSEF, près de $20 \%$ des enseignants cesseraient d'enseigner totalement s'ils en avaient la possibilité et $40 \%$ le ferait «partiellement». Ces souhaits sont très fortement associés au degré d'insatisfaction professionnelle (Maroy, 2002c). Ces phénomènes de « perte d'attractivité » du métier sont documentés à l'échelle européenne par Eurydice (2004) et I'OCDE (2005).

\section{UN CHANGEMENT DE NATURE DU MÉTIER D'ENSEIGNANT ? L'INCIDENCE DES POLITIQUES ANGLAISES CENTRÉS SUR L'ÉVALUATION ET L'ACCOUNTABILITY}

Dans les études françaises ou francophones, les phénomènes d'alourdissement de la charge de travail des enseignants sont surtout rapportées aux évolutions déjà évoquées du public scolaire, de son rapport à l'école, même si les tensions entre les objectifs des politiques éducatives sont aussi considérées (par exemple entre réussite de tous et formation d'une élite sur un principe méritocratique).

Plusieurs études anglo-saxonnes (et notamment anglaises) évoquent également ces facteurs mais insistent de surcroît sur les effets des politiques éducatives elles-mêmes sur le contenu du travail enseignant et leur professionnalisation. L'incidence des politiques n'est d'ailleurs pas seulement considérée en termes de charge de travail mais aussi en terme de contenu du métier et d'autonomie professionnelle. L'orientation de ces études doit bien entendu être rapportée au radicalisme relatif des politiques menées en Angleterre et au Pays de Galles. On y assiste en effet à des changements relativement importants (centralisation du curriculum, développement important de l'autonomie managériale des établissements, instauration de mécanismes de quasi-marché, contractualisation et évaluation externe des établissements, évaluation des enseignants) qui affectent profondément les modes de régulation du système (Whitty et al., 1994 ; Maroy, 2004c) mais aussi le travail enseignant lui-même et le fonctionnement des établissements.

Les études anglaises ayant mis en avant l'hypothèse d'une déqualification des enseignants et d'une «prolétarisation » des enseignants sont relativement 
anciennes. Opposés aux analyses "fonctionnalistes" qui promeuvent une professionnalisation des enseignants, basé sur le modèle des "professions ", plusieurs auteurs d'inspiration marxiste ont ainsi souligné dès les années 1980 l'évolution opposée vers une «prolétarisation » des enseignants. Ozga et Lawn (1981) vont ainsi montrer que la nature du travail enseignant devient plus étroitement contrôlée et les programmes plus précisément définis (en termes d'objectifs comportementaux à atteindre, de stratégies d'enseignement à suivre, de réponses attendues des éléves). L'expertise pédagogique que l'enseignant met en œuvre dans sa classe a été captée par les experts pédagogiques et les spécialistes ministériels en matière de programme et d'évaluation. Enfin, ils mettent également l'accent sur l'accroissement du travail, son intensification: classes plus chargées, tâches supplémentaires (administration, surveillance, etc.).

Cependant, des études plus récentes, dans des optiques théoriques différentes, insistent également sur l'impact des réformes récentes sur la nature du travail enseignant en Angleterre.

Pour Osborn et al. (2000) et Mc Ness et al. (2003), les politiques anglaises récentes (teintées de managérialisme et de marchandisation) tendent à mettre l'accent sur l'accountability (reddition de comptes), le besoin d'élever et de prédéfinir des schools standards et les «normes d'accomplissement " des élèves ; il en résulte une pression à la " performativité " de l'activité enseignante et apprenante. Leurs études auprès de professeurs du secondaire (Encompass Project) comme du primaire (Pace Project) montrent des évolutions significatives et des pressions accrues sur les enseignants.

Pour les enseignants du primaire, «il y a eu un renforcement du cadre pédagogique au travers de la mise en place d'un programme national très prescriptif, prolongé par des initiatives ultérieures concernant l'alphabétisation linguistique et arithmétique. La pression des évaluations externes nationales, et l'influence de la mise en place d'objectifs tant pour les individus que pour les établissements scolaires a aussi affecté le travail des enseignants du primaire en Angleterre pendant les années 1990, créant un contexte de classe qui a été de façon croissante défini de l'extérieur » (Mc Ness et al., 2003, p. 247-248, notre traduction). II en a résulté une tendance à dévaluer les compétences pédagogiques professionnelles des enseignants dès lors que le curriculum était supposé pouvoir "être transmis " de façon relativement aisée, en suivant seulement des étapes prescrites et un découpage didactique prédéfini.

Une tension existe dès lors entre cette orientation prédominante et la conception pédagogique partagée par les enseignants, en raison de leur formation initiale et de leur expérience, qui envisage l'éducation et la scolarisation comme un processus interactif et social dans lequel ils ont un rôle affectif et social clé à tenir pour accompagner et soutenir l'apprentissage des élèves.

Deux types de conséquences en ont résulté sur les identités et pratiques des enseignants du primaire. Tout d'abord, sur le plan identitaire, beaucoup d'enseignants ressentent un sentiment de fragmentation identitaire lié aux tensions entre les attentes officielles (accent sur les compétences techniques et managériales) et leurs conceptions personnelles du métier accentuant l'importance des dimensions "affective et émotionnelle" dans l'activité d'enseignement. Cette tension alimente un sentiment de déqualification et déprofessionnalisation. Cependant, chez d'autres enseignants, on assiste au développement d'un nouveau professionnalisme (Mc Ness et al., 2003, p. 248). Certains enseignants expérimentent de nouvelles marges de manœuvre et ont le sentiment d'être revalorisé et requa- 
lifié même si le curriculum est davantage centralisé et contraint. Cette dernière réaction est liée tant à la biographie personnelle des enseignants (on la retrouve surtout parmi les nouveaux professeurs) qu'à la culture et au climat de l'école.

Ensuite, les réformes poussent à un travail collégial et collectif plus important (Mc Ness et al., 2003, p. 249) et surtout formellement obligatoire. Cependant, ces collaborations peuvent "vraiment émaner de la base " ou bien être dérivées d'une imposition managériale top down (10). Dans ce dernier cas, il en résulte surtout de la «paperasse " de sorte que les enseignants ne voient plus là dedans qu'une distraction par rapport à leur vrai boulot qui est d'enseigner dans la classe.

En définitive, prévaut un sentiment que les priorités sont fixées de l'extérieur. II en résulte un sentiment de perte d'autonomie et d'accomplissement personnel. Selon les auteurs de l'étude Pace, on peut parler d'un tournant dans le climat d'enseignement qui se fonde moins sur l'engagement éthique et la confiance que sur une base contractuelle, de sorte que le travail d'enseignement et d'apprentissage se focalise de plus en plus sur le domaine académique au détriment du domaine socio-affectif et socio-éducatif : «II y a des indications empiriques claires d'un changement de climat passant d'une convention basée sur la confiance à un contrat basé sur la fourniture de services éducatifs susceptibles de rencontrer les demandes externes et des objectifs économiques. La pression temporelle, la demande croissante de planifier, évaluer, atteindre des objectifs définis de l'extérieur génère un environnement dans lequel, pour les élèves comme pour les enseignants, le domaine affectif a été réduit au bénéfice du domaine académique et scolaire " (Mc Ness et al., 2003, p. 249, notre traduction)

Des observations auprès d'enseignants du secondaire (à la faveur du projet Encompass (11)), montrent des décalages et tensions importantes entre les orientations politiques et celles des enseignants. Les enseignants anglais vivent des tensions très vives (plus qu'en France ou au Danemark) entre leur engagement à l'égard des dimensions affective et éducative de leur métier (pastoral and affective dimensions) et la nécessité de faire atteindre par les élèves les objectifs académiques prédéfinis. "Pour les enseignants, l'inclination professionnelle à s'engager plus fortement dans la composante affective de leur travail est en tension avec le modèle managérial promu par les politiques éducatives qui cherche à être "efficace" en relation avec des mesures de résultats d'élèves, définies en rapport avec des finalités strictement académiques " (Mc Ness et al., 2003, p. 255, notre traduction). «Un accent sur la reddition de comptes a renforcé les demandes administratives par rapport à leur travail, alors que la préoccupation de réaliser les objectifs (targets) pour soi-même ou pour les élèves, a réduit le temps et l'espace nécessaire pour une approche plus satisfaisante de la pédagogie » (Ibid.). D'où le titre d'un des articles de ces auteurs: "Is the Effective Compromising the Affective? 》

La montée des nouvelles politiques éducatives en Angleterre et au pays de Galles depuis l'Education Reform Act de 1988, a également inspiré à S. J. Ball un important article sur les transformations de la signification du métier et de l'acte d'enseignement (Ball, 2003). II insiste sur la montée de nouvelles formes de régulation (" un nouveau mode de régulation moins visible, une sorte d'auto-régulation contrôlée à distance " (Ball, 2003, p. 217) qui se présentent sous les formes d'une accentuation de l'autonomie et de la liberté. II s'interroge sur leurs effets sur " ce que cela signifie d'enseigner et d'être enseignant ", sur les relations et les subjectivités dans le champ éducatif. Ball s'intéresse donc moins aux changements de structures qu'aux processus de régulation des relations et des sub- 
jectivités. Dans une filiation foucaldienne, il cherche à montrer les implications éthiques et professionnelles de nouvelles "disciplines " et " technologies " que les politiques anglaises récentes mettent en place (" disciplines" comme la compétition, la survie, l'efficience, les targets, la réussite, la comparaison, les " audits" d'établissements, les évaluations externes, etc).

L'introduction de ces technologies et disciplines, la diffusion d'une nouvelle éthique de la compétition et de la "performativité ", très différente de l'ancienne éthique du jugement et de la coopération, contribuent à développer un sentiment croissant "d'inauthenticité " ou de "schizophrénie " parmi les enseignants. Ceux-ci se trouvent coincés entre leurs éthiques professionnelles et la nécessité de les sacrifier partiellement pour faire face aux demandes croissantes de «performances visibles ". Il y a des sentiments de "colonisation " de leurs pratiques, et de " déprofessionnalisation " qui s'accroissent. Cependant, S. J. Ball reconnaît aussi qu'il peut y avoir ambivalence de la part des enseignants, car ce nouveau climat normatif peut aussi signifier plus positivement un sentiment d'empowerment et de professionnalisation pour d'autres enseignants. La nouvelle éthique de la performativité recouvre en effet la possibilité pour l'individu de développer un « soi triomphant ».

De surcroît, les réformes engendrent pour Ball une intensification du temps de travail des enseignants, et des contradictions apparaissent pour les enseignants entre les activités de premier ordre (faire la classe, etc.) et des activités de second ordre (effort et temps consacré pour rendre compte de ce que l'on fait, pour construire des systèmes d'évaluation, pour collecter des données, et participer à des "évènements " ou des « mises en scène " managériales au sein des écoles, visant à construire des bonnes images organisationnelles (management of impression). Cela renvoie à la nécessité croissante pour les établissements ou les enseignants de produire des sortes d'artefacts, ce que Ball appelle des « fabrications ", soit la production d'une représentation de soi (comme individu ou organisation) au travers de «des documents à visée performative (performative texts) ». (Ball, 2003, p. 224). Ces derniers (rapports d'activités, bilans de pratiques, etc.) sont produits en réponse aux multiples demandes de reddition de compte et d'évaluation émanant de diverses autorités éducatives. Elles sont ambivalentes et paradoxales, car elles visent à la fois à "éluder " l'évaluation, tout en produisant des effets de soumission du seul fait qu'on s'est soumis à son exercice. Cela ne peut qu'accentuer les sentiments d'inauthenticité, de perte de sens et de schizophrénie (entre ce qu'on fait et ce qu'on croit) et une tendance à la transformation de "l'âme de l'enseignant " (teacher soul).

\section{SYNTHĖSE INTERMÉDIAIRE}

De façon assez générale en Europe, au-delà de spécificités nationales, les enseignants connaissent des évolutions significatives de leur métier et une intensification de leur charge de travail qui tient à la complexification de l'exercice de leur métier et à la diversification de leurs charges. Cependant ces changements ne touchent pas au même degré tous les enseignants, et on a vu que la complexification du travail était sans doute plus forte dans les établissements " difficiles " ou dans les classes difficiles d'autres établissements, ce qui se retraduit dans leurs satisfactions professionnelles. 
Par ailleurs, si pour l'essentiel ces évolutions du travail sont imputables aux transformations du public scolaire de l'école, elles sont aussi encouragées, de façon variable selon les pays et contextes scolaires, par la nature des réformes et des politiques éducatives. En Angleterre en particulier, les réformes menées depuis une bonne décennie, basée sur un mixage du modèle de l'État évaluateur et du quasi marché ont conduit à renforcer de nouveaux modes de régulation basés sur l'évaluation, la définition d'objectifs standards, la " responsabilisation " des établissements locaux et de leurs enseignants. Ces politiques infléchissent outre la charge de travail, la nature du métier dont les composantes proprement affectives et éducatives tendent à diminuer. L'ethos des professionnels et leur rapport au métier et à l'école pourraient changer et évoluer d'une logique de l'engagement à une logique de contrat. Paradoxalement d'ailleurs, sur base des travaux de Hargreaves, on pourrait avancer que la surcharge de travail vient partiellement de la volonté des enseignants de préserver et de rester fidèle à leur ethos professionnel antérieur. C'est parce que les enseignants tiennent encore à prodiguer une attention au développement des élèves, au-delà de leur attention aux performances strictement scolaires et au-delà des nouvelles charges administratives qui leur incombent, que la surcharge se fait sentir.

Une telle hypothèse pourrait d'ailleurs être prolongée à partir des travaux récents du groupe de "clinique de l'activité " d'Yves Clot (2005). Les chercheurs associés à ce courant analysent " l'activité " au-delà du prescrit et de la tâche et montrent que non seulement il y a plusieurs manières de répondre aux prescriptions, mais que le « réel de l'activité » est plus large que la seule "activité réalisée ", embrassant "ce qu'on ne fait pas, ce qu'on a voulu faire et ce qu'on n'a pas pu faire, ce qu'on a fait un temps et qui continue d'habiter les situations présentes, ce qu'on n'a pas renoncé à faire sans pour autant y parvenir ". L'activité de l'enseignant en situation, les potentialités ou les empêchements qu'il y rencontre, se situent ainsi au carrefour de différentes dimensions - personnelle, impersonnelle, transpersonnelle, interpersonnelle - du métier. Or justement, les nouvelles prescriptions en termes d'obligation de résultats peuvent tendre à " empêcher " certaines activités de la part de l'enseignant et à en prescrire d'autres, alors que par ailleurs l'ethos professionnel de l'enseignant l'invite à en développer d'autres encore. D'où au final, un sentiment d'aliénation, de tension ou de surcharge.

\section{RÉSISTANCES ET RÉFORMES : POURQUOI LES ENSEIGNANTS « RÉSISTENT-ILS » AUX TRANSFORMATIONS DU MÉTIER ?}

Dans cette dernière partie, nous voudrions enfin nous interroger sur les différentes pistes d'explication qui peuvent être avancées pour rendre compte de la tiédeur, du manque d'enthousiasme voire de la résistance que les enseignants semblent opposer, au moins pour une partie d'entre eux, aux réformes qui sont supposées être faites pour les aider à mieux faire face aux transformations de l'institution scolaire et de leur métier. Ainsi, face aux transformations du public scolaire, à la montée de l'hétérogénéité sociale dans les établissements et classes, face à la transformation des rapports à la culture scolaire ou à l'autorité qui se font jour parmi les jeunes, face à la " nécessité » d'améliorer la qualité des systèmes éducatifs, nombre d'États ont conduit des politiques visant à faire évoluer les enseignants et leurs compétences. Des rhétoriques politiques dites de professionnalisation du métier d'enseignant, le souci de forger une politique de 
« développement professionnel " des enseignants se sont concrétisés de façon variable dans des politiques de réforme de la formation initiale et continuée des enseignants, et/ou dans les réformes des métiers de l'inspection et de l'accompagnement pédagogique des enseignants. (Eurydice, 2001; Tardif \& Lessard, 2004 ; Lang, 1999 \& 2004). Ces réformes s'inspirent souvent du modèle du praticien réflexif (Tardif et al., 1998 ; Cattonar \& Maroy, 2000 ; Lang, 1996 ; Paquay, 1994). Or ces politiques de "professionnalisation " sont rarement soutenues et portées par le milieu enseignant lui-même mais davantage par des experts en pédagogie et les gouvernements.

Face à ces réformes, on assiste souvent à des attitudes réservées voire opposées d'acteurs comme les organisations syndicales, dès lors qu'on leur propose, même contre changement des conditions salariales, d'appliquer le modèle du praticien réflexif, en insistant par exemple sur la nécessité du travail collectif, qu'on instaure une formation continue obligatoire, ou encore que l'implication dans l'établissement, la responsabilisation par rapport à son évaluation est rendue obligatoire. Par exemple, les organisations syndicales enseignantes belges ont revendiqué " un moratoire pédagogique " et une pause des réformes. Ils ont obtenu d'ailleurs une " consultation " des enseignants, et depuis lors le pouvoir politique fait souvent valoir et entendre la nécessité d'obtenir l'adhésion des enseignants aux objectifs gouvernementaux et aux réformes (Van Campenhoudt et al., 2004). Cette méfiance par rapport aux politiques opérationnalisant tout ou partie du modèle du praticien réflexif se retrouve d'ailleurs en France dans la période récente où les organisations syndicales se sont largement déclarés opposées aux conclusions du rapport Thélot (2004) notamment en ce qui concerne la révision des "modalités de service" des enseignants (voir Le Monde du 14 octobre 2004) (12)

Comment peut-on rendre compte de cette méfiance à l'égard des politiques dites de professionnalisation?

\section{Une question de génération et de socialisation au métier ?}

Un premier type d'explication renvoie à la socialisation des enseignants, en particulier des anciens, qui, formés à un modèle de professionnalité antérieur (le modèle du "maître instruit " entre autres) résisterait aux nouveaux modèles (modèle du pédagogue et du praticien réflexif). Les résistances s'expliqueraient ainsi par des traits liés à la culture professionnelle des enseignants, qui seraient d'une part plutôt « individualistes " (Hargreaves, 1993; Huberman, 1993), d'autre part "relativement conservateurs" sur le plan pédagogique, attachés aux modèles pédagogiques auxquels ils ont été formés. Ce serait particulièrement le cas en France, où les débats dans les médias autour des paradigmes pédagogiques à soutenir (plus constructiviste ou plus transmissif) et autour des modes de formation initiale des enseignants ont été assez vifs, ce qui pourrait être dû au type de conception du métier, prégnante en France par comparaison à d'autres pays. Une étude comparée menée par Mac Ness et al. (2003) semble en effet confirmer qu'en France, la conception du travail enseignant au secondaire, est plus académique qu'en Angleterre ou au Danemark.

Face à cette tendance au maintien de modèles anciens, la solution serait alors de renforcer les politiques de formation, notamment à l'endroit des jeunes enseignants, en faisant le calcul que le changement identitaire des enseignants finira par opérer, par contagion lente, surtout parmi les jeunes générations, si on poursuit et on améliore la formation des enseignants et leur entrée dans le métier. 
Une telle explication trouve d'ailleurs un début de confirmation dans le travail de Rayou et van Zanten (2004), qui, à partir d'une enquête qualitative par entretiens, voient apparaître des "nouveaux enseignants " dont les attitudes à l'égard du métier et des réformes, du nouveau modèle de professionnalité seraient relativement changées par rapport à leurs aînés. Ainsi, ces enseignants sont plus enclins à considérer l'idée qu'il faut prendre les élèves tels qu'ils sont, ils sont aussi plus demandeurs de concertation sur la discipline dans les collèges difficiles, moins "honteux" et plus enclins à avouer leurs échecs ou leurs faiblesses en classe, plus disposés à explorer des réponses au cas par cas à leurs problèmes.

Cependant, une telle explication est sans doute incomplète, car elle fait l'impasse sur les politiques qui peuvent donner de bonnes raisons aux enseignants de ne pas s'enthousiasmer pour les réformes qu'on leur propose.

\section{Malaise collectif et résistance à la déprofessionnalisation}

Si les insatisfactions professionnelles peuvent être fort variables selon les conditions concrètes (classes, établissements) dans lesquelles on enseigne (Maroy, 2002), on pourrait penser que le malaise enseignant ne touche pas tous les enseignants au même degré et que dès lors l'essentiel du problème des enseignants se concentre dans les contextes et établissements difficiles. Pour le résorber, il s'agirait dès lors d'améliorer leurs conditions de travail ou de les compenser par des conditions salariales spécifiques, de favoriser leur mobilité et leurs perspectives de carrière, ou plus fondamentalement de combattre la ségrégation scolaire et les établissements "ghetto". Cette interprétation est cependant partielle et peut dès lors induire des politiques insuffisantes.

En effet, le malaise enseignant ne touche pas que les enseignants dont les conditions d'enseignement sont les plus difficiles. Le malaise est vécu collectivement et semble toucher le "corps " enseignant de façon plus diffuse, quel que soit le type d'établissement dans lequel on enseigne et ce dans de nombreux pays. En effet, dans nombre d'enquêtes d'opinion, les enseignants semblent ressentir un sentiment de "perte de statut ", de dévalorisation relative de leur profession (Tardif et al., 1998 ; Eurydice, 2003 ; Lang, 1999). En Belgique, les enseignants sont peu "optimistes " concernant leur avenir et ce quel que soit le type d'établissement où ils enseignent (Maroy, 2002c).

Même si, sur base d'enquêtes d'opinion faites dans la population dans son ensemble, ce sentiment paraît parfois non fondé (13) (Eurydice, 2003 ; Québec, 2004), il nous semble quant à nous que ce sentiment est un symptôme plus large d'un vécu et d'un mouvement de " déprofessionnalisation " du corps enseignant, qui est lié à des évolutions sociales d'ensemble mais qui est aussi favorisé par certaines politiques éducatives.

La question de la professionnalisation ou de la déprofessionnalisation du corps enseignant a été assez largement discutée par nombre de travaux (Bourdoncle, 1991 \& 1993 ; Lessard \& Tardif, 1996 ; Lang, 1999; Maroy \& Cattonar, 2002). Différentes thèses s'opposent. Alors que certains travaux - de nature normative ou prescriptive - insistent sur la nécessité de développer la formation et le développement professionnel des enseignants pour renforcer leur professionnalisme (Paquay et al., 1998), d'autres, ancrés dans une tradition critique, affirment qu'on assiste à l'inverse à une forme de "prolétarisation " des enseignants (Ozga \& Lawn, 1981), ou à tout le moins de réduction de leur fonction à un corps d'exécutants. Enfin, des positions intermédiaires et plus nuancées sont tenues 
(Bourdoncle 1991; Lessard \& Tardif, 1996 ; Maroy \& Cattonar, 2002). Dans ces débats, il nous semble quant à nous, que le groupe professionnel enseignant voit sa position politique et symbolique de négociation se fragiliser dans de nombreux pays (Maroy, 2004c), et que sa capacité à défendre son autonomie professionnelle est, à des degrés divers, relativement effritée, de telle sorte qu'on peut avancer que le risque, sinon la réalité de la déprofessionnalisation n'est pas une illusion de l'esprit (14).

Plusieurs facteurs sociaux ou politiques se conjuguent dans ce processus d'effritement de l'autonomie professionnelle du groupe enseignant et de sa capacité collective de défense de cette autonomie: le développement général du niveau d'éducation des parents; la perte de rareté relative des diplômes et qualifications des enseignants, du secondaire notamment; la concurrence d'autres professionnels de l'éducation ou de la culture (Lang, 1999 ; Duru-Bellat \& Henriot-van Zanten, 1992). Les politiques éducatives par ailleurs ont tendu, à des degrés variables selon les pays, à limiter les marges de manœuvres individuelles et collectives des enseignants dans l'exercice de leur métier: comme par exemple l'introduction, en Belgique et en France, de possibilités de recours ou d'avis des parents dans les décisions d'évaluation ou d'orientation des conseils de classe (Maroy \& Cattonar, 2002) ; l'introduction d'un curriculum et/ou d'objectifs pédagogiques beaucoup plus prescriptifs et contraignants (par exemple en Angleterre ou en Australie), ou encore la mise en place d'outils d'évaluation des résultats des établissements et du travail des enseignants, associés à des procédures de responsabilisation et d'imputation de comptes (Broadfoot, 2000 ; Ball, 2003). Ces effets propres des politiques ont été particulièrement forts en Angleterre, et ont produit notamment des sentiments de "déprofessionnalisation " comme on l'a indiqué plus haut (Osborn et al., 2000).

Mais en outre, on peut constater que des « injonctions paradoxales " tendent à être adressées aux enseignants, notamment en Angleterre ou en Belgique, dans la mesure où d'un côté on leur demandait de devenir " des professionnels " autonomes et réflexifs, alors qu'au même instant, les politiques tendaient à cadrer davantage l'exercice de leur activité professionnelle (Maroy, 2002b ; Ball, 2003).

Dans ces conditions, la capacité collective des enseignants de défendre ou de renforcer l'autonomie professionnelle et le statut professionnel et matériel du groupe tend à être affaiblie et on peut avancer l'hypothèse, que le sentiment de dévalorisation de "la place des enseignants dans la société » exprimé par les enseignants dans les enquêtes d'opinion est une manifestation indirecte et travestie de cet état de choses. Et les enseignants n'ont peut-être pas tort, car le malaise collectif vis-à-vis de leur perte de statut social et professionnel trouve quelques fondements dans les études que nous avons évoquées sur les conditions de travail et d'emploi tout comme sur le cadrage de leur action par certaines des politiques récentes, quand bien même leur autonomie professionnelle individuelle peut encore être forte.

On peut dès lors se demander s'il ne s'agit pas là d'une des sources majeures des attitudes d'ambivalence, de retrait ou de résistance des enseignants face aux réformes. C'est en tous cas l'hypothèse que nous ferions par rapport aux enseignants belges, qui récemment ont demandé via leur organisations syndicales "un moratoire pédagogique ", c'est-à-dire un moratoire des réformes pédagogiques initiées par le gouvernement. Les résistances aux réformes ne suscitent pas des oppositions, uniquement ou seulement, parce que les enseignants seraient individualistes, conservateurs et rétifs au changement de leurs pratiques par «nature» ou «culture professionnelle» (Maroy, 2004). Les 
"réformes " soulèvent au minimum le doute voire l'opposition parce ce qu'elles pourraient renforcer dans certains pays une perte d'autonomie dans les pratiques professionnelles individuelles, un cadrage organisationnel et managérial de leur agir par les chefs d'établissements, voire plus largement un alourdissement et une complexification de leurs tâches et charges de travail par des charges gestionnaires supplémentaires liés notamment aux impératifs d'évaluation et d'imputation de résultats qui tendent à se développer (Maroy \& Cattonar, 2002 ; Maroy 2002b).

II y a donc un enjeu professionnel considérable pour les enseignants dans les politiques de modernisation des systèmes scolaires en cours dans de nombreux pays. Cet enjeu devrait inciter à trouver des voies de négociation des réformes en cours et leur issue dépendra bien entendu tant des attitudes et stratégies adoptées par les pouvoirs publics que celles prises par les organisations syndicales ou les associations professionnelles enseignantes.

Contraintes organisationnelles et difficultés d'application des nouveaux modèles de professionnalité : l'exemple du travail collectif

Les politiques de professionnalisation et les nouveaux modèles de professionnalité enseignante (comme celui du praticien réflexif) engagent on l'a vu une forte valorisation du "travail collectif ". Cela s'est d'ailleurs d'ores et déjà retraduit dans la définition du travail "prescrit " aux enseignants dans un certain nombre de pays (voir supra). En France, le rapport Thélot (2004) allait dans ce sens, puisqu'il recommandait de changer les "modalités de service " pour favoriser la présence des enseignants dans l'établissement, et au-delà la concertation entre enseignants et plus largement le travail collectif. Ce dernier, on le sait, semble en effet relativement peu développé, notamment en France (Barrère, 2002b) ou en Belgique francophone (Dupriez, 2003)

Voyons alors les différentes explications qui ont été avancées pour rendre comple de ce rail, en Belyique el en France.

L'explication la plus paresseuse a été d'incriminer " l'individualisme enseignant ", qui est parfois considéré comme un trait quasi naturalisé de la culture enseignante. Une telle explication est largement insuffisante car il apparaît que les enseignants ne se manifestent pas en principe contre le travail collectif (Barrère, 2002b; Maroy, 2004), en particulier chez les «nouveaux » enseignants (Rayou \& van Zanten, 2004).

La faiblesse du travail collectif doit être mis en relation, de façon plus pertinente, avec la forme même de l'organisation scolaire qui n'incite guère à collaborer, notamment en raison de la structure "cellulaire " du travail en classe (Tardif \& Lessard, 1999; Hargreaves, 1993) mais aussi du fait que l'organisation matérielle, spatiale, temporelle offre rarement des conditions propices à ce travail collectif (Dupriez, 2003). (15) Par ailleurs, la dimension organisationnelle est aussi invoquée comme facteur explicatif lorsque le faible développement du travail collectif est associé au faible enjeu que représenterait l'établissement pour les enseignants et au faible pouvoir dont disposerait le chef d'établissement vis-à-vis des enseignants. Cependant, une telle situation peut être très variable selon les pays (notamment dans les pays qui décentralisent peu ou prou la gestion du personnel enseignant aux établissements comme l'Angleterre, la Hollande ou la Belgique) et elle évolue dans le sens d'une plus grande autonomie et pouvoir des chefs d'établissement (voir supra). Par ailleurs, cette thèse a été contredite pour la France par Barrère (2002b) qui montre que les enjeux de répartition des classes et des horaires pouvaient fonder un pouvoir informel important des directions. 
Plus que les formes organisationnelles, ce sont aussi les normes collectives de travail au sein des établissements (relevant de ce que J.-D. Reynaud appelle la régulation autonome) qui sont à considérer. Ces normes de régulation professionnelle locale peuvent conduire les enseignants à protéger et renforcer l'autonomie de chaque individu dans sa classe et plus largement privilégier le statu quo au développement d'un travail collectif. Ainsi Meyer et Scott (1983), insistent sur les normes et stratégies "d'évitement des conflits", de "voilement des activités" (chacun ignore ou fait mine d'ignorer ce que fait l'autre) ou de " minimisation des incidents " qui se conjuguent dans les établissements pour réguler le rapport mutuel que les enseignants entretiennent souvent entre eux. (voir aussi Dupriez, 2003 ; Maroy, 1992). Barrère (2002b) montre aussi que les acteurs n'ont pas forcément intérêt à sortir du statu quo et à rechercher activement le développement d'une approche plus collective de leur travail, quand bien même ils ne sont pas opposés sur le principe et plutôt ouverts à ce travail collectif. En effet, dans le système français, les enseignants dont la «réputation» de «tenir sa classe " est moyenne et assez bonne, n'ont pas intérêt à dévoiler leurs pratiques et expériences dans la mesure où leur bonne image dans l'établissement est suffisante pour obtenir des avantages dans le jeu de répartition des classes à l'intérieur de l'établissement ou dans les espoirs de mobilité professionnelle horizontale. De même le chef d'établissement peut craindre les effets d'un développement d'espaces de débat et négociation dans l'école. Par ailleurs la "sociabilité professionnelle affinitaire ", largement partagée par les enseignants, est un obstacle à des collaborations qui seraient rendues obligatoires, liées aux fonctions et aux tâches, dont l'initiative et la régulation seraient aussi confiées au personnel d'encadrement dans les établissements.

Mais plus fondamentalement selon Barrère (2002b), une des raisons du faible développement du travail collectif tient surtout à ce que les objets de ce travail collectif ne portent pas directement ou suffisamment sur les enjeux professionnels les plus cruciaux pour les enseignants : soit tout ce qui peut affecter la gestion de la classe, ce qui concerne la mise en place des conditions de l'apprentissage et d'un ordre scolaire. Si le travail collectif affecte et porte sur ces enjeux, on peut penser que l'intérêt qu'il représente pour les enseignants augmentera. Cette thèse trouve d'ailleurs des éléments de confirmation dans les travaux de Kherroubi et van Zanten (2000) qui montrent que le travail collectif se développe plus aisément dans les "établissements difficiles " où les enseignants trouvent dans le développement de stratégies collectives face aux incivilités des réponses pertinentes face à ce qui leur importe.

Ces éléments d'analyse permettent de préciser les conditions qui doivent être prises en compte au niveau organisationnel pour convaincre les enseignants d'adhérer et de mettre en œuvre les modèles de professionnalité qui font une large place au travail collectif.

Pour que cette virtualité s'actualise, il ne suffit pas, comme le suggère le rapport Thélot (2004), de procéder à une redéfinition formelle de « modalités de service" (présence obligatoire au-delà du temps d'enseignement dans l'école) couplée à une accentuation de l'autonomie de gestion des établissements (évaluation des personnels par le chef d'établissement et définition contractuelle et négociée des "modalités de service " au niveau de l'établissement). II faut encore que l'organisation et l'orientation du travail collectif porte sur des domaines jugés importants par les enseignants eux-mêmes, faute de quoi il sera considéré comme une charge supplémentaire, un facteur d'intensification du travail qui accentue les tensions et freine l'investissement dans ce qui reste considéré comme le cœur du 
métier. Une prescription du travail collectif par les normes formelles ou la direction des établissements risque dans ces conditions d'augmenter d'autant la tendance au "malaise enseignant " et à accentuer le sentiment de déprofessionnalisation.

\section{CONCLUSIONS}

Nous avons d'abord montré les traits structurels et relativement permanents du travail enseignant, liés notamment à la prégnance de la forme scolaire. Après avoir souligné l'évolution des «modèles de professionnalité " qui peuvent servir de base à la construction de l'identité professionnelle, nous avons aussi développé les évolutions du travail prescrit, montrant qu'on assiste à une diversification et à un accroissement du nombre de tâches demandées formellement aux enseignants dans la plupart des pays européens investigués par Eurydice. Au niveau du travail réel, la littérature semble de plus s'accorder sur un constat d'intensification et de complexification du travail des enseignants. L'intensification se marque moins par un allongement de la durée du travail, que par un alourdissement et une extension des tâches à réaliser, et par une complexification du travail en classe qui constitue le cœur du métier. Simultanément, on peut se demander si on n'assiste pas aussi à une différenciation assez nette des conditions d'exercice du métier selon le type d'établissement où l'on enseigne.

Cependant, cette intensification du travail ne doit pas seulement être rapportée à des évolutions du public scolaire ou à la ségrégation existant entre établissements scolaires. En Angleterre, en tous cas, elle doit aussi être rapportée aux politiques scolaires visant à développer l'évaluation et l'accountability au sein des écoles et du système. Plus précisément, les enseignants vivent des sentiments de déprofessionnalisation ou de tensions entre leurs orientations normatives et celles des politiques dans la mesure où la dimension « affective » et éducative du métier tend à devoir être mise en veilleuse, au profit d'une logique d'enseignement plus instrumentale. La surcharge de travail peut aussi être paradoxalement liée dans ces contextes aux tentatives des enseignants de satisfaire simultanément les demandes officielles et leurs propres conceptions du métier.

Enfin, nous nous sommes interrogés sur les raisons de la tiédeur, pour ne pas dire de la résistance des enseignants, face à des réformes qui sont supposés les aider à faire face à un environnement scolaire en profonde transformation. Le problème est-il seulement celui d'une résistance au changement lié à des cultures professionnelles dépassées, à une socialisation à des modèles de métier dépassés. Est-ce seulement une affaire de génération enseignante ? Le malaise enseignant face aux politiques de professionnalisation et aux changements du "métier » enseignant, nous semble plus profondément lié à des formes de retrait ou de résistance face aux réformes, lorsqu'elles accentuent la déprofessionnalisation des enseignants. Plus particulièrement, nous nous sommes penchés brièvement sur la question spécifique du travail collectif des enseignants, que toutes les politiques scolaires européennes tendent à encourager: au-delà de l'individualisme enseignant, la littérature montre que la faiblesse de ce travail collectif peut être aussi liée aux conditions organisationnelles dans lesquelles il est encouragé et au type de problème qu'il permet ou non de prendre à bras le corps. Ainsi, lorsque le travail collectif ou les concertations portent sur des questions dont l'interdépendance aux enjeux directs du travail de l'enseignant est claire à 
ses yeux (enjeux internes à la classe, ou plus largement à l'ordre dans l'établissement), il semble que la participation des enseignants puisse être fortement favorisée.

En définitive, le malaise enseignant est lié à l'intensification et à la complexification du travail qui se développe à des degrés variables selon les différents segments du champ scolaire. Mais le malaise existe aussi à un niveau plus collectif et peut être entretenu par les politiques, de sorte que l'acteur enseignant tend dans ce cas à « résister " au changement, faute de pouvoir suffisamment se le réapproprier ou l'orienter en fonction des enjeux qu'il juge prioritaire à partir de son activité, de son expérience et de son ethos professionnel.

\author{
Christian Maroy \\ maroy@anso.ucl.ac.be \\ Université catholique de Louvain \\ Groupe interfacultaire de recherche \\ sur les systèmes d'éducation et de formation (GIRSEF)
}

\title{
NOTES
}

(1) Une première version de ce texte a fait l'objet d'une conférence " libre examen " donnée dans le cadre du Programme incitatif de recherche en éducation et formation (PIREF) le 24 mars 2005 à Paris et a été publiée comme Cahier de recherche en éducation et formation, $\mathrm{n}^{\circ} 42$, disponible sur le site Internet du Groupe interfacultaire de recherche sur les systèmes d'éducation et de formation (GIRSEF) de l'université catholique de Louvain. L'auteur remercie Hughes Draelants, Marie Duru-Bellat, et des arbitres anonymes de la Revue francaise de pédagogie pour leurs commentaires sur cette nouvelle version du texte, dont il assume seul la responsabilité.

(2) On retrouve à des degrés divers trace de ces trois composantes dans divers rapports « prospectifs " nationaux ou internationaux sur les transformations nécessaires de l'école. Par exemple dans le rapport Thélot (2004), mais il n'est pas difficile d'en retrouver des traces dans nombre d'argumentaires des réformes menées dans les différents pays européens (Maroy, 2004) ou nord-américains. Voir par exemple le projet de " Contrat stratégique pour l'éducation "en Communauté française de Belgique (http://www.contrateducation.be ; consulté le 7 avril 2006) ; ou encore le document québécois, La gouverne de l'éducation : priorités pour les prochaines années (Québec. 2004).

(3) En ce qui concerne la littérature anglophone, il est basé méthodologiquement sur une recherche bibliographique systématique des bases de données anglo-saxonne Eric, Currents Contents Educational Management Abstracts, en combinant les termes clés suivants: Workload, Work load, Teaching Load, charge de travail, Teacher/Teachers, enseignant, enseignant au primaire, Elementary Education/Primary Education/Primary School/Primary Schools, Secondary School/Secondary Schools, Reform, Educational Change ". Pour la littérature francophone, le compte rendu est prioritairement centré sur les ouvrages et articles qui se sont interrogés de façon directe sur la question de l'évolution du travail enseignant. Ont été privilégiés les ouvrages proposant une recherche empirique originale, une approche conceptuelle distincte ou une synthèse de la question. Des ouvrages se centrant sur des questions connexes (comme la profession enseignante, l'identité, la formation etc.) sont aussi mobilisés dans la mesure où ils abordent indirectement ou secondairement la question du travail enseignant. Vu le point de vue adopté ici, et les contraintes de volume, nous ne pourrons leur rendre complètement justice.

(4) D'où la tendance à « professionnaliser " le travail enseignant c'est-à-dire à l'organiser selon ce que les sociologues anglosaxons appellent le modèle professionnel. Les métiers qui s'or- ganisent selon ce modèle tendent en effet à déléguer aux professionnels eux-mêmes la régulation de leurs activités. Plutôt que d'être contrôlés par voie hiérarchique, sur base des règles de l'organisation (bureaucratie), l'activité est contrólée par le professionnel et ses pairs. Mais les enseignants qui travaillent dans des bureaucraties professionnelles, tendent à être simultanément des exécutants soumis au respect de normes bureaucratiques.

(5) D'autres blocs beaucoup moins importants quantitativement ef qualitativement concernent le rapport aux collègues (supervision de stagiaires, échanges pédagogiques entre collègues, etc.), la formation et le développement professionnel, et enfin la participation des enseignants à des tâches organisationnelles (comites, coordonnateur pedagogique, organisation syndicale). Voir Tardif \& Lessard, 1999, p. 141, tableau 3.8.

(6) Les " experts " en sciences de l'éducation, les « pouvoirs organisateurs " de l'enseignement, les « intervenants pédagogiques ", les mouvements pédagogiques, les syndicats et les associations de parents

(7) «Modèle de professionnalité enseignante » que nous entendons comme l'ensemble des pratiques, d'attitudes et compétences attendues et jugées nécessaires à l'exercice de la profession enseignante, selon une définition inspirée de Lang, 1996 ; Lessard et al., 1991; Paquay et al., 1996.

(8) Cette conception d'un « établissement mobilisé " ne fera pas ici l'objet d'une analyse spécifique. Signalons cependant que nombre de travaux de chercheurs ont porté sur des cas d'établissements concrétisant, parfois depuis longtemps, ces orientations (voir Dubet et al., 1989 ; van Zanten et al., 2002 ; Dutercq \& Derouet, 2004)

(9) En 2002, les quinze pays de l'Union européenne, trois pays de l'AELE et douze pays candidats à l'adhésion.

(10) Des initiatives récentes du gouvernement anglais (paie au mérite, «threshold barrier for career advencement») pourrait selon les auteurs de l'étude PACE accentuer encore le sentiment de division entre les collègues et rendre plus difficile les initiatives autonomes de travail collectif, par les enseignants eux-mêmes.

(11) Le projet Encompass est une étude comparative des attitudes des élèves à l'égard de la scolarisation au secondaire dans trois pays : Angleterre, France, Danemark.

(12) On sait que ce rapport proposait d'expliciter davantage et d'insister sur la pluralité des missions des enseignants (enseigner mais aussi éduquer), de revoir les modalités de service, en ren- 
dant la présence dans l'établissement obligatoire pour les nouveaux enseignants, moyennant d'ailleurs une revalorisation salariale. Cette présence était en effet considérée par le rapport comme un moyen de favoriser un établissement plus " mobilisé " et travaillant de façon plus concertée et coopérative.

(13) La profession enseignante reste une des professions dans lequel le public a le plus confiance.

(14) Un tel point de vue se fonde sur une approche « néo wébérienne " des groupes professionnels et de la professionnalisation (Maroy \& Cattonar, 2002). La professionnalisation est alors envisagée comme un processus politique entre un groupe professionnel,
l'État, les usagers et d'autres groupes professionnels, dont l'enjeu porte sur l'autonomie professionnelle, le statut matériel et symbolique du groupe et de son activité professionnelle. Cela signifie qu'une " profession " désigne un groupe professionnel qui a réussi à contrôler de façon relativement autonome, mais jamais totale, son champ de travail, les modes d'accès et de formation au métier, la régulation de son activité. Cette approche est clairement opposée à l'approche « fonctionnaliste "

(15) La situation belge et française peut à cet égard est assez dissemblable de celle qui prévaut dans certains établissements finlandais, suédois ou australiens, si on en juge par certains compte rendu de voyages pédagogiques.

\section{RÉFÉRENCES BIBLIOGRAPHIQUES}

Alt M. N. ; Kwon J. \& Henke R. R (1999). Teachers and Teaching. Results from the Schools and Staffing Survey. Washington [DC] : National Center for Education Statistics, $25 \mathrm{p}$.

BALL S. J. (2003). « The teacher's soul and the terrors of performativity ». Journal of Éducation Policy, vol. $18, n^{\circ} 2$, p. $215-228$.

BARRĖRE A. (2002). Les enseignants au travail. Paris : L'Harmattan.

Barrère A. (2002b). "Pourquoj les enseignants ne travaillent-ils pas en équipe?"Sociologie du travail, vol. 44, n 4, p. 481-497.

Baumaro M. (2004). "Autant d'horaires que de statuts ", Le Monde de l'éducation, $n^{\circ} 329$, p. 26-27.

BAutier É. et al. (1995). Travailler en banlieue : la culture de la professionnalité. Paris : L'Harmattan.

BAUTIER É \& RocheX J.-Y (1997). « Apprendre : des malentendus qui font la différence $»$. In J.-P. Terrail, La scolarisation de la France: critique de l'état des lieux. Paris : La Dispute, p. 105-112.

BERGER I. (1979). Les instituteurs d'une génération à l'autre. Paris : PUF.

BIDWELL C. E. (1965). "The school as a formal organization". In J. G. March (éd.), The Handbook of Organizations. Chicago : Rand McNally.

BLIN J.-F. (1997). Représentations, pratiques et identités professionnelles. Paris : L'Harmattan.

BOURDONCLE R. (1991). "La professionnalisation des enseignants: analyses sociologiques anglaises et américaines ". Revue française de pédagogie, $n^{\circ} 94$, p. 73-92.

BOURDONCLE R. (1993). «La professionnalisation des ensejgnants: les limites d'un mythe ". Revue française de pédagogie, $n^{\circ} 105$, p. 83-119.

BROADFOOT P. (2000). « Un nouveau mode de régulation dans un système décentralisé : l'État évaluateur ». Revue française de pédagogie, $n^{\circ} 130$, p. 43-55.

CASAlFIORE S. \& DE KETELE J.-M. (2002). « Exercer le métier d'enseignant en classe: les fonctions qui structurent l'activité quotidienne des enseignants ». In C. Maroy (éd), Les écoles d'enseignement secondaire et leurs enseignants. Bruxelles : De Boeck, p. 209-242.

Cattonar B. (2002). " Identités professionnelles et conceptions du métier chez les enseignants du secondaire libre en Communauté française de Belgique ". In C. Maroy (éd.), Les écoles d'enseignement secondaire et leurs enseignants. Bruxelles : De Boeck, p. 171-208.

Cattonar B. (2005). L'identité professionnelle des enseignants du secondaire en Belgique francophone. Thèse de doctorat: sociologie, université catholique de Louvain.

Cattonar B. \& Maroy C. (2000). «Rhétorique du changement du métier d'enseignant et stratégie de transformation de l'institution scolaire". Education et sociétés: revue internationale de sociologie de l'éducation, $n^{\circ} 6$, p. 21-42.

ChAPOULIE J.-M. (1974). « Le corps professoral dans la structure de classe ". Revue française de sociologie, vol. XV, $\mathrm{n}^{\circ} 2$, p. 155-200.

CHAPOULIE J.-M. (1979). « La compétence pédagogique des professeurs comme enjeu de conflits". Actes de la recherche en sciences sociales, $n^{\circ} 30$, p. 65-85.

ChaPOULIE J.-M. (1987). Les professeurs de l'enseignement secondaire: un métier de classe movenne. Paris: Éd. de la MSH.

Chatel É. ; Rochex J.-Y. \& Roger J.-L. (1996). « Transformations du second degré et métier d'enseignant ». In C. Grémion \& R. Fraisse (dir.), Le service public en recherche: quelle modernisation ? Paris : La Documentation Française, p. 329-338.

CHENU A. (2002). « Les horaires et l'organisation du temps de travail ", Economie et statistiques, $n^{\circ} 352-353$, p. 151167.

Clot Y. [dir.] ; Litim M. ; Prot B. ; Roger J.-L. \& Ruelland D. [collab.] (2005). Enjeux du travail et "genres " professionnels dans la recomposition en cours des métiers de la fonction publique. Le cas des professeurs d'enseignement secondaire et du personnel soignant de gérontologie. Paris : CNAM : Laboratoire de psychologie du travail et de l'action.

CORNET J. (1999). "Souffrance de classe ». Le Ligueur, n 1.

Cousin O. (1998). L'efficacité des collèges: sociologie de l'effet établissement. Paris: PUF.

Delvaux B. : Dourte F. \& Verhoeven M. (1996). Transformations du métier d'enseignant : pratiques et représentations des intervenants. Louvain : Université catholique de Louvain; Centre de recherches interdisciplinaires pour la solidarité et l'innovation sociale (CERISIS).

DEMAILLY L. (1987). "La qualification ou la compétence professionnelle des enseignants". Sociologie du travail. vol. XXIX, n' 1, p. 59-69. 
Demailly L. (1991). Le collège: crise, mythes et métiers. Lille : Presses universitaires de Lille.

DuBAR C. (1996). La socialisation : construction des identités sociales et professionnelles. Paris: A. Colin.

Dubet F. (1991). Les lycéens. Paris : Éd. du Seuil.

DUBet F. (2002) Le déclin de l'institution. Paris : Éd. du Seuil.

Dubet F. ; Cousin O. \& Guillemet J.-P. (1989). “ Mobilisation des établissements et performances scolaires". Revue française de sociologie, vol. XXX, $n^{\circ} 2$, p. 235-256.

Dubet F. \& Martucelli D. (1996), À l'école: sociologie de l'expérience scolaire. Paris: Ed. du Seuil.

DUPRIEZ V. (2002). "Entre tradition et transformations, que révèle l'analyse des établissements scolaires? " In C. Maroy (dir.), L'enseignement secondaire et ses enseignants. Bruxelles: De Boeck, p. 55-90.

DUPRIEZ V. (2003). " De l'isolement des enseignants au travail en équipe : les différentes voies de construction de l'accord dans les établissements ". Cahiers de recherche en éducation et formation, $\mathrm{n}^{\circ} 23$

Dupriez V. \& VANDENBerghe V. (2004). "L'école en Communauté française de Belgique: de quelle inégalité parlons-nous? "Cahiers de recherche en éducation et formation, $n^{\circ} 20$.

Duru-Bellat, M. \& HenRiot-Van Zanten A. (1992). Sociologie de l'école. Paris: A. Colin; "La profession enseignante», p. $139-158$.

DUTERCQ Y. (1993). Les professeurs, Paris : Hachette.

Duterce Y. \& DeRouet J.-L. [dir.] (2004). Le collège en chantier. LyOn : INRP.

EAsthope C. \& EASTHOPE G. (2000). «Intensification, Extension and Complexity of Teacher's Workload ". British Journal of Sociology of Education, vol. $21, n^{\circ} 1$, p. $43-$ 58.

EURYDICE (2001). La profession enseignante en Europe: profil, métiers et enjeux, rapport l. La formation initiale et la transition vers la vie professionnelle des enseignants du niveau secondaire inférieur général. Bruxelles: Eurydice.

EURYDICE (2002). La profession enseignante en Europe : profil, métiers et enjeux, rapport II. L'offre et la demande d'enseignants au niveau du secondaire inférieur général. Bruxelles : Eurydice.

EURYDICE (2003a). La profession enseignante en Europe: profil, métiers et enjeux, rapport III. Conditions de travail et salaires. Bruxelles : Eurydice.

Eurydice (2003b). Communiqué de presse. La profession enseignante en Europe: profil, métiers et enjeux, rapport III. Conditions de travail et salaires. Bruxelles: Eurydice.

EURYDICE (2004). La profession enseignante en Europe : profil, métiers et enjeux, rapport IV. L'attractivité de la profession enseignante $a u X X^{e}$ siècle. Bruxelles: Eurydice.

FRANCE : MINISTÉre dE L'ÉdUCATION NATIONALE: DIRECTION DE L'ÉVALUATION ET DE LA PROSPECTIVE (1996). Connaissance des enseignants. Paris : MEN (Éducation et formations, $\left.n^{\circ} 46\right)$.

GoRARD S \& SMITH E. (2004). " An international comparison of equity in education systems ". Comparative Education, vol. $40, n^{\circ} 1$, p. $15-28$.
Hargreaves A. (1993). «Individualism and Individuality: Reinterpreting the Teacher Culture ». In J. W. Little \& M. W. McLaughlin, Teacher's Work. Individuals, Colleagues and Contexts. New York: Teachers College Press.

Hargreaves A. (1994). Changing Teachers, Changing Times: Teacher's Work and Culture in the Postmodern Age. London: Cassel.

HASenfeld Y. \& AbBott A. D. (1992). Human Services as Complex Organisations. Newbury Park [CA] : Sage.

HiRSCHHORN M. (1993). L'ère des enseignants. Paris : PUF.

Huberman M. (1993). "The Model of the independent Artisan in Teachers' Professional Relations ". In J. W. Little \& M. W. McLaughlin, Teacher's Work. Individuals, Colleagues and Contexts. New York: Teachers College Press.

KherRoubi M \& A. VAN ZANTEN (2000). "La coordination du travail dans les établissements "difficiles" : collégialité, division des rôles et encadrement ". Éducation et sociétés: revue internationale de sociologie de l'éducation, $\mathrm{n}^{\circ}$ 6, p. 65-92.

LANG V. (1996). "Professionnalisation des enseignants, conception du métier, modèles de formation". Recherche et formation pour les professions de l'éducation, $\mathrm{n}^{\circ} 23$, p. 9-27.

LANG V. (1999). La professionnalisation des enseignants: sens et enjeux d'une politique institutionnelle. Paris: PUF.

LANG V. (2004). "La profession enseignante en France : permanence et éclatement ". In M. Tardif \& C. Lessard, La profession d'enseignant aujourd'hui : évolutions, perspectives et enjeux internationaux. Laval [Québec]: Presses de l'université Laval, p. 157-172.

LÉGER A. (1983). Enseignants du secondaire. Paris : PUF.

LESSARD C. (1991). " Le travail enseignant et l'organisation professionnelle de l'enseignement: perspectives comparatives et enjeux actuels ». In C. Lessard, M. Perron \& P. W. Bélanger (dir.), La profession enseignante au Québec. Enjeux et défis des années 1990. Québec: Institut québécois de recherche sur la culture, "Documents de recherche ", $n^{\circ} 30,1991$, p. 15-40.

Lessard C. (2000) "Présentation du dossier "Nouvelles régulations et professions de l'éducation" "Éducation et sociétés : revue internationale de sociologie de l'éducation, $\mathrm{n}^{\circ} 6$, p. $5-20$

Lessard G. ; Tardif M. \& Lahaye L. (1991). "Pratiques de gestion, régulation du travail enseignant et nouvelle professionnalité ". In C. Lessard, M. Perron \& P.W. Bélanger (dir.), La profession enseignante au Québec. Enjeux et défis des années 1990, Institut québécois de recherche sur la culture, "Documents de recherche ", $n^{\circ} 30,1991$, p. 69-91.

LESSARD C. \& TARDIF M. (1996). La profession enseignante au Québec (1945-1990): histoire, structures, système, éducation et formation, fondements. Montréal : Presses de l'université de Montréal.

LESSARD C. \& TARDIF M. (2004). " Les transformations actuelles de l'enseignement: trois scénarios possibles dans l'évolution de la profession enseignante ». In M. Tardif \& C. Lessard, La profession d'enseignant aujourd'hui : évolutions, perspectives et enjeux internationaux. Laval [Québec] : Presses de l'université Laval, p. 265-288. 
Mac Ness E. ; Broadfoot P. \& Osborn M. (2003). "Is the Effective Compromising the Affective? "British Educational Research Journal, vol. 29, $\mathrm{n}^{\circ} 2$, p. 243-257.

MARoY C. (1992). " L'école à la lumière de la sociologie des organisations ». Éducation-formation, $n^{\circ} 228$, p. 27-50.

MAROY C. [dir.] (2002). L'enseignement secondaire et ses enseignants. Bruxelles : De Boeck.

MAROY C. (2002b). "Quelle autonomie professionnelle des enseignants? ". Revue internationale d'éducation de Sèvres, $n^{\circ} 30$, p. 41-50.

Maroy C. (2002c) «Contexte de travail, climat d'établissement et satisfaction professionnelle ". In C. Maroy (dir.), L'enseignement secondaire et ses enseignants. Bruxelles : De Boeck, p. 131-170.

Maror C. (2002d). "Conclusion générale ». In C. Maroy (dir.), L'enseignement secondaire et ses enseignants. Bruxelles : De Boeck, p. 243-266.

MAroY C. (2004). "Régulation et évaluation des résultats des systèmes d'enseignement ". Politiques d'éducation et de formation, vol. 11, $\mathrm{n}^{\circ} 2$, p. 21-36.

MAROY C. (2004b). "Le modèle du praticien réflexif à l'épreuve de l'enquête en Belgique ». In M. Tardif \& C. Lessard (dir.), La profession d'enseignant aujourd'hui : évolutions, perspectives et enjeux internationaux. Laval [Québec] : Presses de l'université Laval.

MARoY C. (à paraître). École, régulation et marché : une analyse de six espaces scolaires en Europe. Paris: PUF.

MAROY C. \& CATTONAR B. (2002). "Professionalisation ou déprofessionalisation des enseignants? Le cas de la Communauté française de Belgique ". Cahiers de recherche en éducation et formation, $n^{\circ} 18$.

Maroy C. \& CATtonar B. (2002b). "L'évolution sociologique des enseignants du secondaire en Belgique francophone ". In C. Maroy (dir.), L'enseignement secondaire et ses enseignants. Bruxelles: De Boeck, p. 93-110.

MARoY C. \& Dupriez V. (2000). «La régulation dans les systèmes scolaires. Proposition théorique et analyse du cadre structurel en Belgique francophone ". Revue française de pédagogie, $\mathrm{n}^{\circ} 130$, p. 73-87.

MEYER J. W. \& SCOTT W. R. (1983). Organizational Environments : Ritual and Rationality. Beverly Hills [CA] : Sage.

Muel-Dreyfus F. (1983). Le métier d'éducateur : les instituteurs de 1900, les éducateurs spécialisés de 1968. Paris : Éd. de Minuit.

NEAVE G. (1988). «On the cultivation of quality, efficiency and enterprise : an overview of recent trends in higher education in Western Europe, 1986-1988». European Journal of Education, vol. 23, $\mathrm{n}^{\circ} 1-2$, p. 7-23.

ORGANISATION POUR LA COOPÉRATION ET LE DÉVELOPPEMENT ÉCONOMIQUE (2005). Teachers Matter: Attracting, Developing and Retaining Effective Teachers. Synthesis Report. Paris : OCDE.

Osborn M. ; Mc Ness E. \& Broadfoot P. (2000). What Teachers Do : Changing Policy and Practice in Primary Education : Findings from the Pace Project. London : Continuum.

OZGA J. \& LAWN M. (1981). Teachers Professionalism and Class : a Study of Organized Teachers. London : Falmer Press.
PAQUAY L. (1994). «Vers un référentiel de compétences professionnelles de l'enseignant ? "Recherche et formation pour les professions de l'enseignement, $n^{\circ} 16, p .7-38$.

Paquay L. ; Altet M. ; Charlier E. \& Perrenoud P. [éd.] (1996). Former des enseignants professionnels: quelles stratégies ? Quelles compétences ? Bruxelles: De Boeck.

PerRenoud P. (1993). "Formation initiale des maîtres et professionnalisation du métier ». Revue des sciences de l'éducation, vol. XIX, n 1, p. 59-76.

Perrenoud P. (1994). La formation des enseignants entre théorie et pratique. Paris : L'Harmattan.

Perron M. ; Lessard C. \& Bélanger P. W. (1993). " La professionnalisation de l'enseignement et de la formation des enseignants : tout a-t-il été dit ? ". Revue des sciences de l'éducation, vol. XIX, $n^{\circ} 1$, p. 5-32.

PEYRONIE H. (1998). Instituteurs : des maitres aux professeurs d'école. Paris : PUF.

QuÉBEC: CONSEIL SUPÉRIEUR DE L'ÉDUCATION (2004). Un nouveau souffle pour la profession enseignante. Avis au ministre de l'éducation. Québec : CSE.

RAYOU P. \& VAN ZANTEN A. (2004). Enquête sur les nouveaux enseignants : changeront-ils l'école ? Paris : Bayard.

ROCHEX J.-Y. (1995) «Enseignants en banlieue ou enseignants de banlieue? ". In É. Bautier et al., Travailler en banlieue : la culture de la professionnalité. Paris: L'Harmattan, p. 165-255.

SCHÖN D. (1983). The Reflective Practitioner. New York: Basic Books.

TANGUY L. (1991). L'enseignement professionnel en France: des ouvriers aux techniciens. Paris : PUF.

TARDIF M. \& LESSARD C. (1999). Le travail enseignant au quotidien: expérience, interactions humaines et dilemmes professionnels. Bruxelles: De Boeck.

TARDIr M. \& LessanD C. [dir.] (2004). La profossion d'onscignant aujourd'hui. Évolutions, perspectives et enjeux internationaux. Laval [Québec] : Presses de l'université Laval.

TARDIF M. ; Lessard C. \& Gauthier C. [éd.] (1998). Formation des maitres et contextes sociaux. Paris : PUF.

THÉLOT C. (2004). Pour la réussite de tous les élèves. Rapport de la Commission du débat national sur l'avenir de l'école présidée par Claude Thélot. Paris : La Documentation Française.

Van Campenhoudt L. ; Franssen A. ; Gaelle H. ; Van Espen A. ; LEJEUNE A. \& HUYNEN P. (2004) La consultation des enseignants du secondaire. Rapport élaboré pour la Commission de Pilotage. Bruxelles : ministère de la Communauté française.

VAN ZANTEN A. (2001). L'école de la périphérie: scolarité et ségrégation en banlieve. Paris : PUF.

van Zanten A. ; Grospiron M.-F. ; Kherroubi M. \& Robert A. (2002) Quand l'école se mobilise. Paris : La Dispute.

VINCENT G. [dir.] (1994). L'éducation prisonnière de la forme scolaire? Lyon: Presses universitaires de Lyon.

WhitTY G. ; POWER S. \& HALPIN D. (1998). Devolution \& Choice in Education. The School, the State and the Market. Philadelphia : Open University Press. 